ARTICLE

DOI: $10.1038 / \mathrm{s} 41467-018-04453-9$

\title{
Hypoxia induces senescence of bone marrow mesenchymal stem cells via altered gut microbiota
}

Junyue Xing 1,2,3, Yongquan Ying ${ }^{4}$, Chenxi Mao5 ${ }^{5}$, Yiwei Liu'1,2, Tingting Wang ${ }^{1,2}$, Qian Zhao ${ }^{1}$, Xiaoling Zhang ${ }^{1,2}$, Fuxia $\mathrm{Yan}^{3} \&$ Hao Zhang ${ }^{1,2,3}$

Systemic chronic hypoxia is a feature of many diseases and may influence the communication between bone marrow (BM) and gut microbiota. Here we analyse patients with cyanotic congenital heart disease (CCHD) who are experiencing chronic hypoxia and characterize the association between bone marrow mesenchymal stem cells (BMSCs) and gut microbiome under systemic hypoxia. We observe premature senescence of BMSCs and abnormal Dgalactose accumulation in patients with CCHD. The hypoxia that these patients experience results in an altered diversity of gut microbial communities, with a remarkable decrease in the number of Lactobacilli and a noticeable reduction in the amount of enzyme-degraded Dgalactose. Replenishing chronic hypoxic rats with Lactobacillus reduced the accumulation of Dgalactose and restored the deficient BMSCs. Together, our findings show that chronic hypoxia predisposes BMSCs to premature senescence, which may be due to gut dysbiosis and thus induced D-galactose accumulation.

\footnotetext{
${ }^{1}$ State Key Laboratory of Cardiovascular Diseases, Fuwai Hospital, National Center for Cardiovascular Diseases, Chinese Academy of Medical Sciences and Peking Union Medical College, Beijing 100037, China. ${ }^{2}$ Key Laboratory of Cardiac Regenerative Medicine, National Healthy Commission, Fuwai Hospital, Beijing 100037, China. ${ }^{3}$ Center for Pediatric Cardiac Surgery, Fuwai Hospital, CAMS\&PUMC, Beijing 100037, China. ${ }^{4}$ Department of Thoracic and Cardiovascular Surgery, Taizhou Hospital, Zhejiang 317000, China. ${ }^{5}$ Department of Thoracic and Cardiovascular Surgery, 1st Affiliated Hospital of Wenzhou Medical University, Zhejiang 325006, China. These authors contributed equally: Junyue Xing, Yongquan Ying, Chenxi Mao. Correspondence and requests for materials should be addressed to H.Z. (email: Drzhanghao@yahoo.com)
} 
S ystematic chronic hypoxia is a pathological state associated with inadequate whole-body oxygen supply. A variety of diseases, including cyanotic congenital heart disease (CCHD), chronic obstructive pulmonary disease, chronic mountain sickness and pulmonary fibrosis, have been extensively reported to be implicated in this process ${ }^{1,2}$. Clinically, the hypoxic condition concomitant with diseases not only exacerbates the process of disease per se but also brings about much more detrimental effects on the body ${ }^{3}$. Exploring the downstream pathophysiological responses to the systematic hypoxia markedly appears to be necessary for the disease cure, especially in the aspects of providing new therapeutic alternatives.

Many clinical studies have focussed on the capacity of bone marrow mesenchymal stem cells (BMSCs) to immunosuppress and tissue regeneration ${ }^{4-6}$. Notably, BMSC infusions have shown promise in ameliorating steroid-resistant acute graft-versus-host disease with prolonged overall survival after allogeneic stem cell transplantation $^{7-9}$. Based on their potential to differentiate into various kinds of cells, BMSCs have been applied as tissue engineering patches/conduits in many clinical trials to treat spinal cord injury $^{10}$, ischaemic stroke ${ }^{11,12}$, myocardial infarction ${ }^{13}, 14$, osteoarthritis ${ }^{15}$ and other diseases. BMSCs are mainly located in the perivascular zone of the bone marrow $(\mathrm{BM})^{16}$. Oxygen is a critical component of the BM niche, and a hypoxic environment in the $\mathrm{BM}$ is generally considered to be necessary for maintenance of normal physiological function and self-renewal of stem cells ${ }^{17}$. However, the hypoxic status in BM can be aggravated in patients experiencing systemic hypoxia and may induce distinctive physiological changes.

Mounting evidence has supported the hypothesis that communications exist between the gut and the $\mathrm{BM}^{18,19}$. The gut microbiota has been shown to regulate haematopoietic stem cell differentiation by impairing BM function in obesity ${ }^{20}$. The gut lumen contains trillions of metabolically active microbes ${ }^{21}$. Both the microbiota and the highly vascularized intestinal mucosa that lies immediately adjacent to the gut lumen are sensitive to chronic hypoxia $^{22}$. Therefore, the chronic profound hypoxia could alter the diversity of gut microbiota and result in the accumulation of gut microbiota-derived metabolites, which might significantly impact homeostasis of the host.

In this study, patients with CCHD were utilized as the human disease model of systemic chronic hypoxia to investigate the associations between the $\mathrm{BM}$ and gut. Intriguingly, we observe premature senescence of BMSCs in CCHD patients, which is implicated in gut dysbiosis and gut microbiota-derived D-galactose accumulation. Moreover, D-galactose accumulation in chronic hypoxic rats is decreased upon administration of moderate amount of Lactobacillus and also deficient BMSCs is restored significantly.

\section{Results}

Patient recruitment. Ninety children undergoing surgical repair for CHD from 2013 to 2016 were enrolled. The exclusion criteria were as follows: age $<6$ months, diarrhoea, receiving antibiotics or hormone therapy within the last 12 weeks, extracardiac anomalies, and chromosomal abnormalities. The patients were divided into a non-cyanotic (NCy) group $(n=45)$ and a cyanotic (Cy) group $(n=45)$. All patient characteristics are summarized in Table 1 . Forty samples were sent for metabolic profiling analysis ( $n=20$ per group), and another 40 samples were utilized for cell culture and phenotype analysis ( $n=20$ per group). Ten samples with adequate numbers of BM were randomly chosen for blood gas analysis ( $n=5$ per group). Among the 90 patients, 40 were chosen randomly for stool $16 \mathrm{~S}$ ribosomal RNA and metagenomic sequencing ( $n=20$ per group).

\section{Table 1 Baseline characteristics of the enrolled patients}

\begin{tabular}{lccc} 
& $\begin{array}{c}\text { Group NCy }(\boldsymbol{n}= \\
\mathbf{4 5})\end{array}$ & Group Cy $(\boldsymbol{n}=\mathbf{4 5})$ & $\boldsymbol{P}$ \\
& & & \\
\hline Age (months) & $23.10 \pm 10.69$ & $21.92 \pm 18.78$ & 0.217 \\
Male (\%) & $48.89 \%$ & $53.3 \%$ & 0.673 \\
Height $(\mathrm{cm})$ & $91.40 \pm 6.33$ & $78.33 \pm 9.38$ & $<0.01$ \\
Weight $(\mathrm{kg})$ & $12.98 \pm 1.69$ & $9.61 \pm 1.72$ & $<0.01$ \\
$\mathrm{Hb}(\mathrm{g} / \mathrm{dl})$ & $12.50 \pm 0.95$ & $16.91 \pm 2.99$ & $<0.01$ \\
Diagnosis & ASD12 VSD 33 & TOF 15 TGA 10 & - \\
& & PAA 9 DORV 11 &
\end{tabular}

ASD atrial septal defect, VSD ventricular septal defect, TOF tetralogy of fallot, TGA transposition of the great arteries, PAA pulmonary artery atresia, DORV double outlet right ventricle

Table 2 Arterial and BM blood gas analysis in the NCy and Cy groups

\begin{tabular}{|lcll} 
& NCy group $(\boldsymbol{n}=\mathbf{5})$ & Cy group $(\boldsymbol{n}=\mathbf{5})$ & $\mathbf{P}$ \\
\hline $\mathrm{SaO}_{2}(\%)$ & $99.13 \pm 0.90$ & $77.18 \pm 5.89$ & $<0.01$ \\
$\mathrm{PaO}_{2}(\mathrm{~mm}$ & $115.90 \pm 8.75$ & $45.38 \pm 5.13$ & $<0.01$ \\
$\mathrm{Hg})$ & & & \\
$\mathrm{SbmO}_{2}(\%)$ & $87.67 \pm 3.06$ & $43.00 \pm 7.94$ & $<0.01$ \\
$\mathrm{PbmO}_{2}(\mathrm{~mm}$ & $51.67 \pm 5.51$ & $26.33 \pm 3.05$ & $<0.01$ \\
$\mathrm{Hg})$ & & \\
\hline & & & \\
\hline $\mathrm{SaO}_{2}$ arterial oxygen saturation, $\mathrm{PaO}_{2}$ arterial blood oxygen partial pressure, $\mathrm{SbmO}_{2}$ bone \\
$\mathrm{marrow}$ oxygen saturation, $\mathrm{PbmO}_{2}$ bone marrow oxygen partial pressure \\
\hline
\end{tabular}

BMSCs from CCHD patients undergo premature senescence. To evaluate the oxygen tension in BM and peripheral blood, the oxygen saturation of the patients with or without CCHD was measured. The partial pressure of oxygen $\left(\mathrm{pO}_{2}\right)$ and oxygen saturation $\left(\mathrm{SO}_{2}\right)$ were significantly reduced in the $\mathrm{BM}$ compared with the peripheral arterial blood in both groups $(51.67 \mathrm{~mm} \mathrm{Hg}$ vs. $115.9 \mathrm{~mm} \mathrm{Hg}$ in the NCy group, $P<0.01 ; 26.33 \mathrm{~mm} \mathrm{Hg}$ vs. $45.38 \mathrm{~mm} \mathrm{Hg}$ in the Cy group, $P<0.01$; Student's $t$-test). The $\mathrm{SO}_{2}$ and $\mathrm{pO}_{2}$ in the $\mathrm{BM}$ from the Cy group $(26.33 \mathrm{~mm} \mathrm{Hg}$ ), however, demonstrated an even greater reduction (by half) than that in the BM from the NCy group ( $51.67 \mathrm{~mm} \mathrm{Hg}, P<0.01$, Student's $t$-test) (Table 2).

To investigate the effect of chronic hypoxia on BMSCs, we cultured BMSCs and assessed the expression of a variety of senescence markers. The morphology and phenotype of BMSCs are shown in Supplementary Figure 1. Expression of p16 $(\sim 1.99$ fold, $P=0.002$, Student's $t$-test) but not p53, p21 or p27 was significantly higher in the Cy group than in the NCy group (Fig. 1a). The 3-[4,5-dimethylthiazol-2-yl]-2,5 diphenyl tetrazolium bromide (MTT) assay showed that BMSCs in the Cy group grew more slowly than those in the NCy group (Fig. 1b). The proportion of cells displaying the protein marker senescenceassociated (SA)- $\beta$-galactosidase was dramatically increased in the Cy group $(\sim 57.95 \%$ vs. $\sim 9.27 \%$ in the NCy group, $P<0.01$, Student's $t$-test). The percentage of quiescent-state cells (G0/G1 phase) in the Cy group was significantly higher than that in the NCy group $(\sim 89.53 \%$ vs. $\sim 74.59 \%, P<0.01$, Student's $t$-test $)$, whereas the percentages of cells in the $S$ and G2/M phases in the Cy group were much lower than those in the NCy group (Fig. 1d). The antiapoptosis assay showed a much greater proportion of BMSCs undergoing early apoptosis (Annexin V+/PI-) ( 4.70\% vs. $\sim 1.80 \%, P<0.01$, Student's $t$-test) and late apoptosis (Annexin $\mathrm{V}+/ \mathrm{PI}+)(\sim 6.50 \%$ vs. $\sim 2.93 \%, P<0.01$, Student's $t$-test $)$ in the Cy group than in the NCy group (Supplementary Fig. 1C), 
a
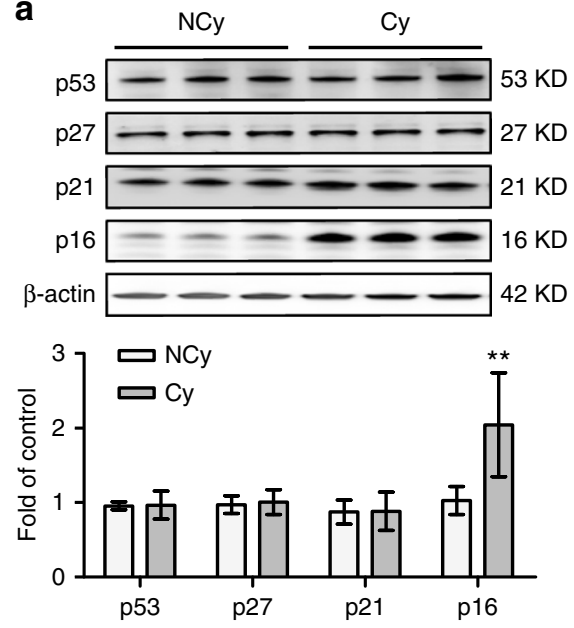

C
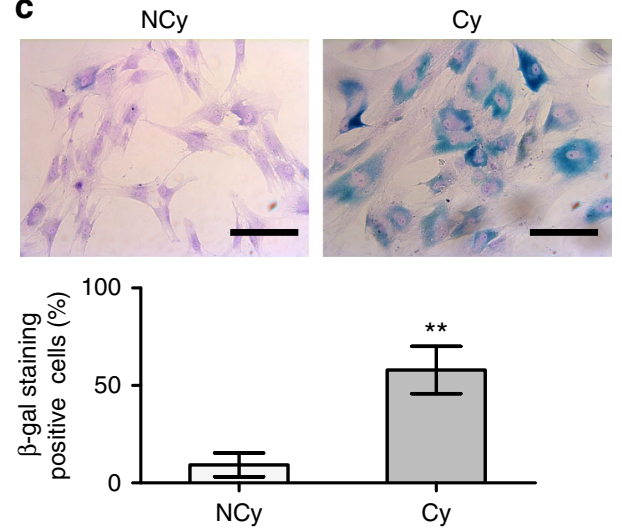

b

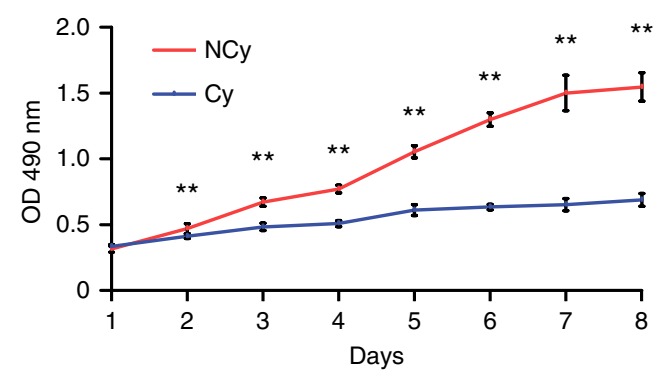

d
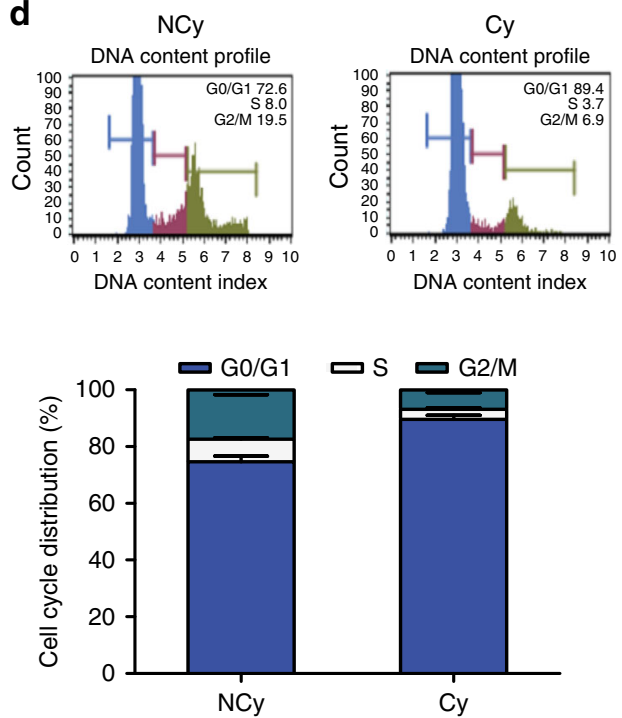

Fig. 1 BMSCs from CCHD patients are predisposed to premature senescence. a BMSCs from non-CCHD (NCy) and CCHD (Cy) patients ( $n=5$ per group) were isolated and cultured to passage 3. Cell lysates were prepared to assess the levels of p53, p27, p21, p16 and $\beta$-actin by western blotting. b Cell numbers were determined by MTT assays at the indicated times. OD optical density. c SA- $\beta$-gal activity was assessed in the cells described in a. BMSCs stained in blue were labelled $\beta$-galactosidase-positive cells. Representative images are shown (upper). Scale bar, $50 \mu \mathrm{m}$. Twenty fields of each section were randomly selected to calculate the ratio of $\beta$-galactosidase-positive cells. $\mathbf{d}$ Cells described in a were collected and subjected to FACS analysis to assess the cell cycle distribution (upper). The percentage of cells in each cell cycle compartment is presented graphically (lower). Data represent the mean \pm SD from three independent experiments, and statistical significance was analysed using Student's $t$-test $\left({ }^{\star \star} P<0.01\right)$

indicating the attenuated antiapoptosis ability of BMSCs from patients with CCHD. Alizarin red staining and an alkaline phosphatase (ALP) activity assay $(\sim 0.10 \mathrm{ng} / \mu \mathrm{l}$ vs. $\sim 0.32 \mathrm{ng} / \mu \mathrm{l}, P<$ 0.01 , Student's $t$-test) revealed impaired osteogenesis potential in the Cy group (Supplementary Fig. 2A, D). In the adipogenic differentiation analysis, the differentiation rate in the Cy group was substantially lower than that in the NCy group ( $\sim 11.37 \%$ vs. $\sim 41.84 \%, P<0.01$, Student's $t$-test) (Supplementary Fig. 2B, E). In the chondrogenic differentiation assay (Supplementary Fig. $2 \mathrm{C}$, F), Type II collagen immunofluorescent staining showed a decreased differentiation rate in the Cy group $(\sim 30.84 \%$ vs. $\sim 51.87 \%, P<0.01$, Student's $t$-test). Taken together, these results suggested that BMSCs from patients with CCHD were predisposed to premature senescence and had impaired multilineage differentiation potential.

Profound hypoxia altered metabolic profiling in BM. To explore the reason of BMSCs premature senescence in patients with $\mathrm{CCHD}$, we employed a gas chromatography time-of-flight mass spectrometry (GC-TOF/MS)-based metabolomics approach to investigate metabolite alterations in the BM. In the total ion current profiles (Supplementary Fig. 3A), 543 valid peaks were identified. The partial least-squares discriminant analysis (PLSDA) and orthogonal partial least-squares discriminant analysis
(OPLS-DA) exhibited a significant separation of clusters between the two groups (Supplementary Fig. 3B-D). A total of 49 metabolites displayed significantly different levels [variable importance in the projection (VIP, computes the influence on $Y$ of every term in the model, is the sum over all model dimensions of the contributions variable influence) $>1$, adjusted $P<0.05$ ], as identified by the OPLS-DA model (Supplementary Table 1). The different metabolites were mainly composed of carbohydrates, amino acids, nucleotides and their intermediate metabolites. The metabolome map revealed that the different metabolites were enriched in glycolysis, gluconeogenesis, the TCA cycle and galactose metabolism pathways (pathway impact value $>0.2$, adjusted $P<$ 0.01; Fig. 2a). These pathways are involved in glycometabolism and amino acid metabolism, which are required for energy production and protein synthesis, respectively.

Moreover, metabolomics highlighted D-galactose, a well-known agent for senescence induction through the production of large amounts of reactive oxygen species (ROS) ${ }^{23}$, as the most relevant metabolite alteration in CCHD (47.07-fold). We also found an extremely high level of the D-galactose metabolic shunt metabolite galactonic acid (30.34-fold) and a low level of galactose-1phosphate (0.433-fold). We tested the D-galactose in peripheral blood and fresh BM supernatant. D-Galactose levels in the Cy group remained higher than those in the NCy group, both in 

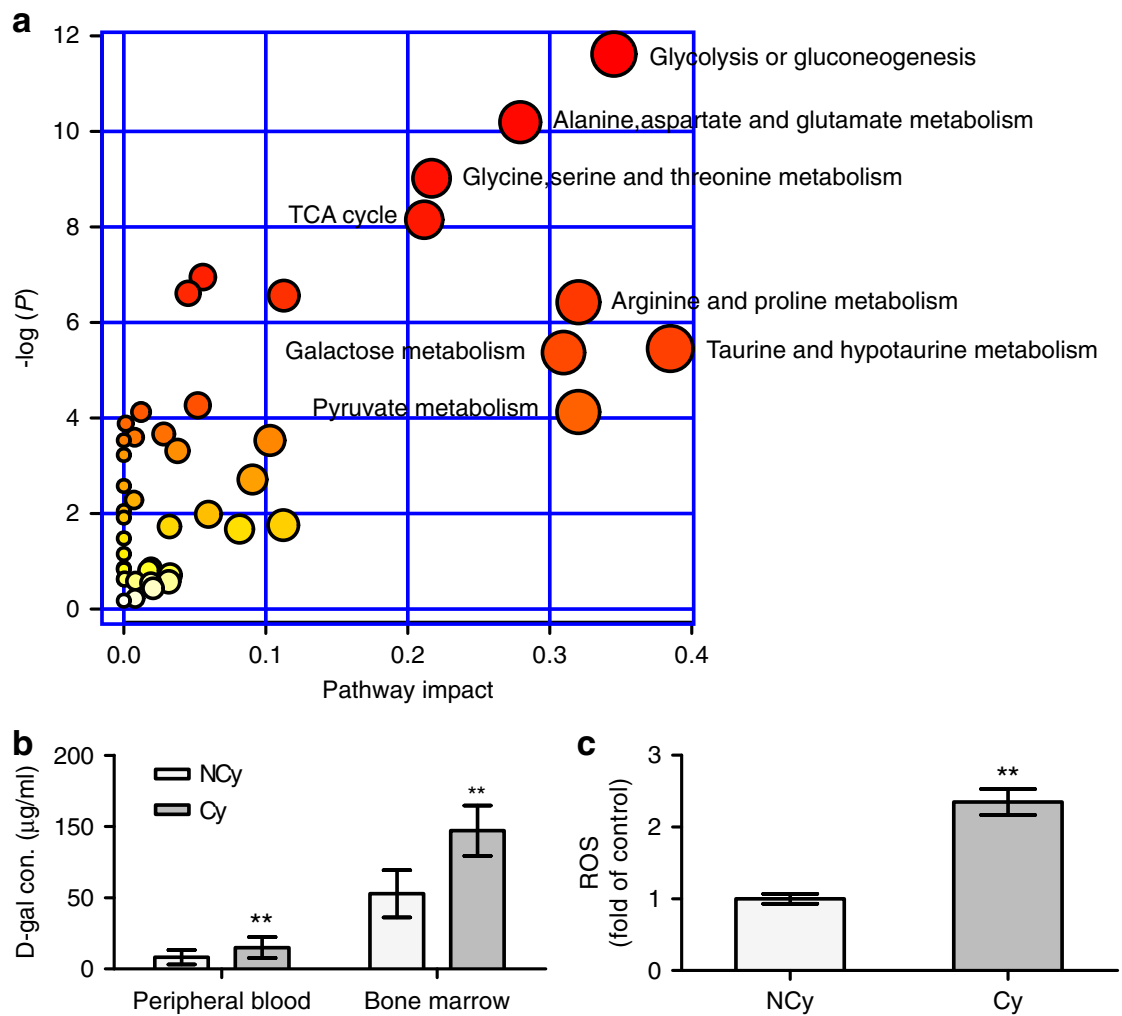

Fig. 2 Profound hypoxia altered metabolic profiling in BM. a Pathway analysis of 49 metabolites identified as being present in the Cy group but not in the NCy group. $x$ Axis represents the pathway impact, and $y$ axis represents the pathway enrichment. Larger sizes and darker colours represent increased pathway enrichment and higher pathway impact values, respectively. $\mathbf{b}$ D-galactose concentrations in the peripheral blood ( $n=22$ per group) and bone marrow ( $n=5$ per group) of patients were analysed. c ROS levels of BMSCs from the NCy and Cy groups were analysed. The data shown are the mean \pm SD from three independent experiments, and statistical significance was analysed using Student's $t$-test ( ${ }^{\star \star} P<0.01$ )

peripheral blood $(\sim 15.0 \mu \mathrm{g} / \mathrm{ml}$ vs. $\sim 8.3 \mu \mathrm{g} / \mathrm{ml}, P<0.01$, Student's $t$-test) and in BM supernatant $(\sim 97.0 \mu \mathrm{g} / \mathrm{ml}$ vs. $\sim 52.9 \mu \mathrm{g} / \mathrm{ml}, P<$ 0.01 , Student's $t$-test) (Fig. 2b). Additionally, the Cy group showed a higher ROS level than the NCy group $(\sim 2.35$-fold, $P<$ 0.01 , Student's $t$-test) (Fig. 2c).

Accumulation of D-galactose induced BMSC senescence. To investigate the association between D-galactose accumulation and premature senescence of BMSCs, BMSCs from the NCy group were cultured in an incubator with low (4\%) or normal $(21 \%) \mathrm{O}_{2}$ (designated Hypo- or Nor-), and the cells were exposed to normal $(50 \mu \mathrm{g} / \mathrm{ml})$ or high $(90 \mu \mathrm{g} / \mathrm{ml})$ concentrations of D-galactose (designated $\mathrm{N}$ - or $\mathrm{H}-$ ). There was no difference between the Nor$\mathrm{N}-\mathrm{Gal}$ and Nor-H-Gal groups in terms of the expression of senescence-related genes (Fig. 3a). Compared with the Hypo-NGal group, the Hypo-H-Gal group presented 1.47-fold higher levels of P16. A two-way analysis of variance (ANOVA) showed that neither hypoxia $(P=0.063)$ nor $D$-galactose $(P=0.181)$ was a statistically significant factor for P16 expression, while interaction of these factors ( $P=0.004$, two-way ANOVA) had a significant effect on the level of P16. The levels of P53, P21 and P27 proteins and $\beta$-actin remained unchanged. Cell proliferation, SA$\beta$-gal activity and multilineage differentiation were significantly attenuated only in the Hypo-H-Gal group (Fig. 3b, c; Supplementary Fig. 2). In the cell cycle assay, the number of cells in G0/ G1 phase also increased in the Hypo-H-Gal group (Fig. 3d), indicating that the cell cycle was arrested by a synergistic combinatorial effect of high D-galactose and hypoxia. In addition, there was a distinct increase in ROS in the Hypo-H-Gal group $(\sim 1.70$-fold) (Fig. 3e) compared with the Nor-N-Gal group. Our findings indicated that D-galactose accumulation and hypoxia induced premature senescence of BMSCs in the NCy group.

Profound hypoxia resulted in a reduction of Lactobacillus. To elucidate the mechanism responsible for D-galactose accumulation, we identified the enzymes that mediate D-galactose metabolism in human BMSCs. The D-galactose metabolism pathway is shown in Supplementary figure $4 \mathrm{~A}$. Western blot analysis showed no significant differences between the NCy and Cy groups in the levels of GALM, GALK1, GALK2, GLB1 and aldose reductase (Supplementary Fig. 4B).

Considering that lactose is the main source of D-galactose and is absorbed into the body via the intestine, $16 \mathrm{~S}$ sequencing was performed and demonstrated marked alterations of the gut microbial communities in the Cy group (Fig. 4). The rarefaction analysis curves for each group were near saturation, suggesting that the sequencing data had a high quality and that very few new species were present (Fig. 4a). Analysis of similarity (ANOSIM) confirmed the significant separation of the groups $(R=0.2, P=$ 0.001 , non-parametric analyses (ANOSIM)) (Fig. 4b), indicating clear differences in microbial composition in the NCy and Cy groups. The top 10 microbes at the phylum (Fig. 4c), family (Fig. 4d) and genus (Fig. 4e) levels are shown and indicate significant variations in the composition of the gut microbiota. Analyses of the microbiota at the phylum level revealed a dominance of Firmicutes, Bacteroidetes and Proteobacteria in both groups and a greater ratio of Firmicutes/Bacteroidetes (2.68-fold, $P$ $=0.001$, Student's $t$-test) in the Cy group than in the NCy group (Fig. 4c). We observed a decrease in the family Lactobacillaceae (0.05-fold, $P=0.024$, metastats) and the genus Lactobacillus (0.05fold, $P=0.016$, metastats) in the Cy group (Fig. $4 \mathrm{~d}-\mathrm{e}$ ). There was 
a
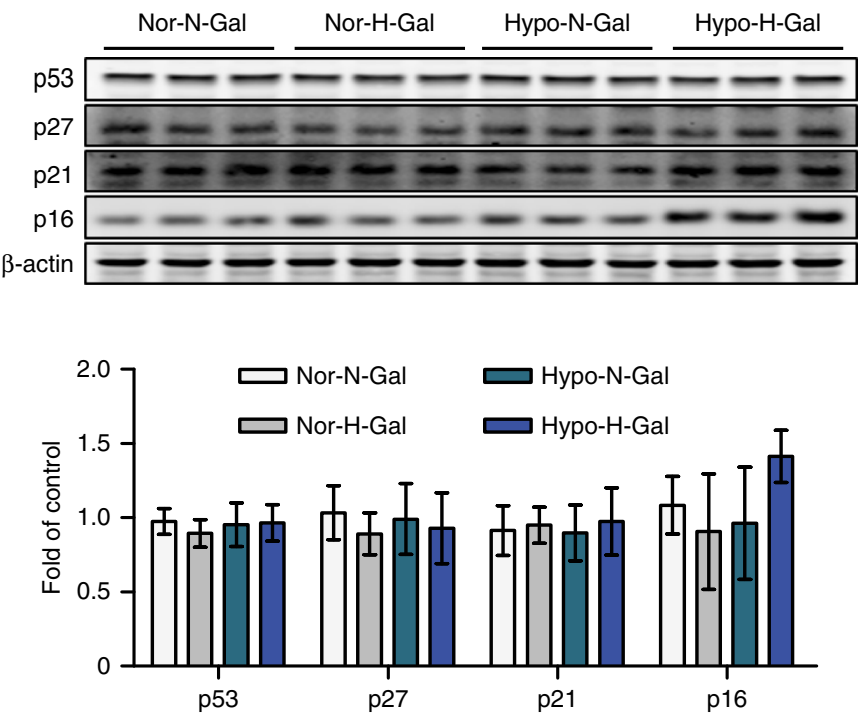

C

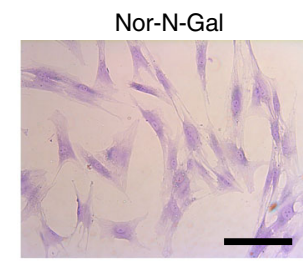

Hypo-N-Gal
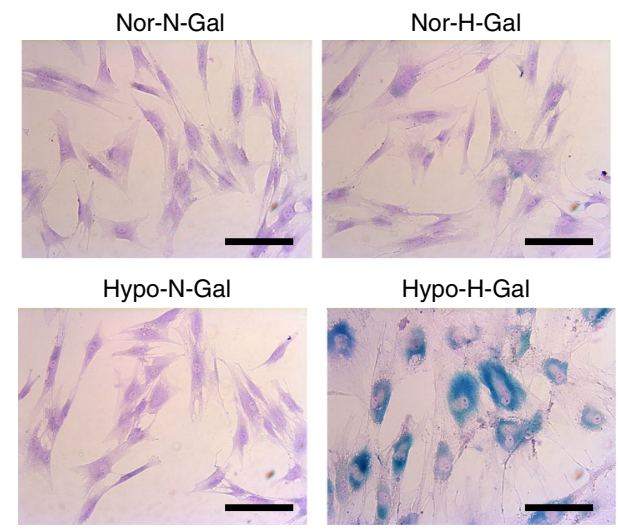

Hypo-H-Gal
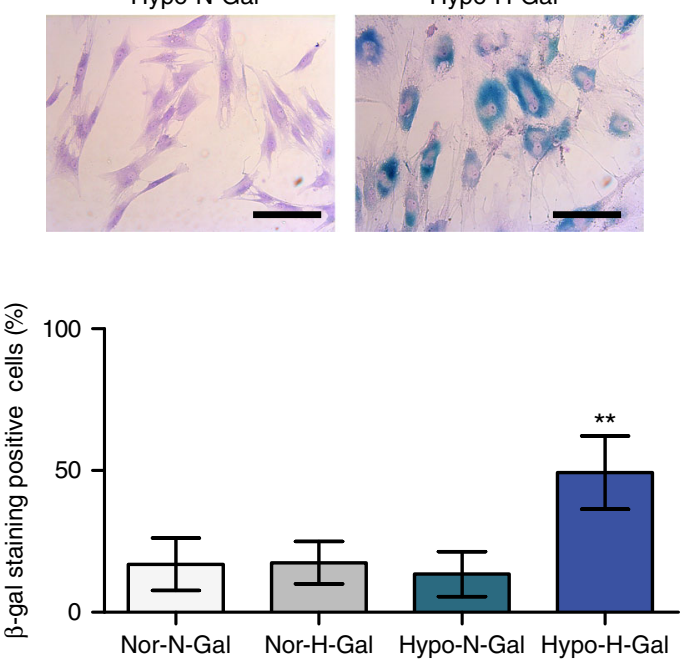

b

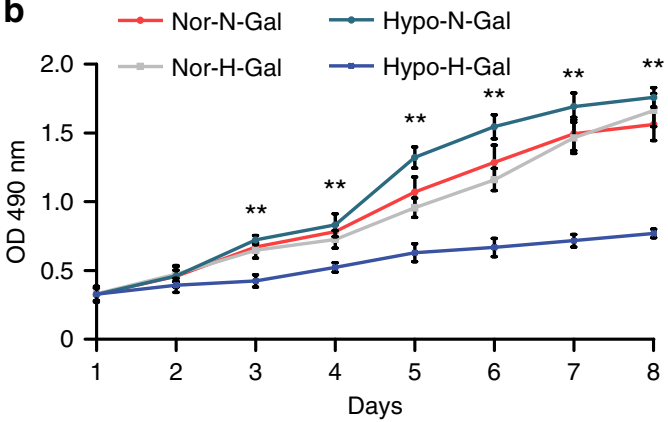

d
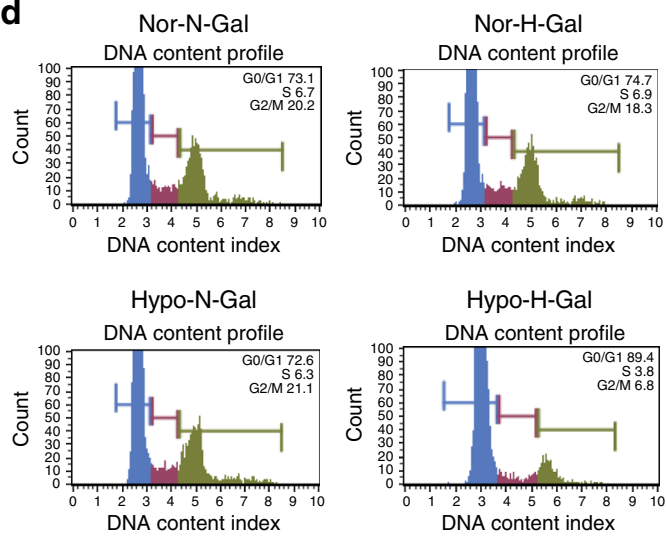

DNA content index

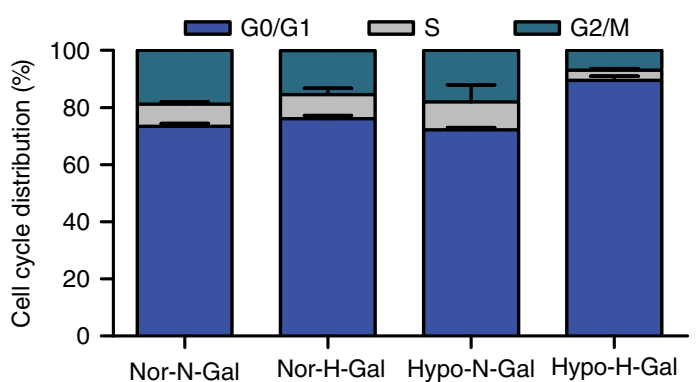

e

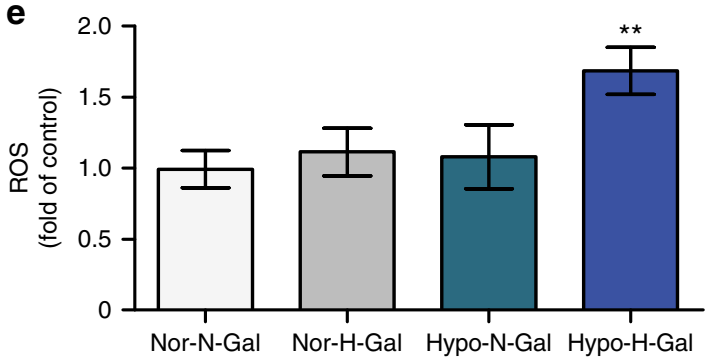

Fig. 3 D-galactose accumulation and hypoxia led to premature senescence of BMSCs. a BMSCs from patients exposed to normal ( $50 \mu \mathrm{g} / \mathrm{ml})$ or high ( 90 $\mu \mathrm{g} / \mathrm{ml})$ concentrations of D-galactose and cultured in a hypoxic $\left(4 \% \mathrm{O}_{2}\right)$ or normoxic $\left(21 \% \mathrm{O}_{2}\right)$ incubator. Samples were designated Hypo- $\mathrm{N}-\mathrm{Gal}$, Hypo- $\mathrm{H}-$ Gal, Nor-N-Gal or Nor-H-Gal accordingly. Cell lysates were subjected to western blot analysis to assess the protein levels of p53, p27, p21, p16 and $\beta$-actin. b Cell numbers were determined by the MTT assay. c The cells described in a were subjected to SA- $\beta$-gal analysis. Scale bar, $50 \mu \mathrm{m}$. $\mathbf{d}$ The cells described in a were subjected to FACS analysis. e Cellular ROS levels were analysed. The data shown are the mean \pm SD from three independent experiments, and statistical significance was analysed by two-way ANOVA $\left({ }^{\star \star} P<0.01\right)$

an increase in Enterobacteriaceae (2.06-fold, $P=0.015$, metastats) but a decrease in Lachnospira (0.33-fold, $P=0.045$, metastats), Ruminococcaceae (0.46-fold, $P=0.014$, metastats) and Faecalibacterium (0.31-fold, $P=0.014$, metastats) in the Cy group. LEfSe analysis showed an increased distribution of Lactobacillus in the NCy group (Fig. 4f). Furthermore, metagenomics sequencing showed that enzymes that degrade D-galactose (lactase (LCT), $P$ $=0.03$; aldose 1 -epimerase, $P=0.03$; GALE (UDP-glucose 4 - epimerase), $P=0.01$; Fisher's exact test with false discovery rate (FDR) adjustment) were decreased in the Cy group (Fig. 4g).

Finally, Pearson correlation analyses (Fig. 4h) showed that the abundance of Lactobacillus in a patient's stool had a negative relationship with the D-galactose concentration in the patient's blood (Pearson correlation coefficients, $R=-0.706, P<0.01$ ).

Collectively, patients with CCHD experienced gut microbiota dysbiosis, especially a reduction in Lactobacillus, which was 
a

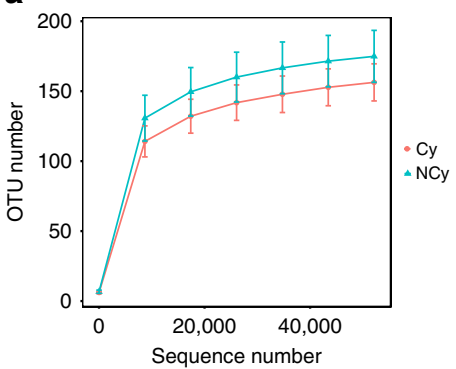

d

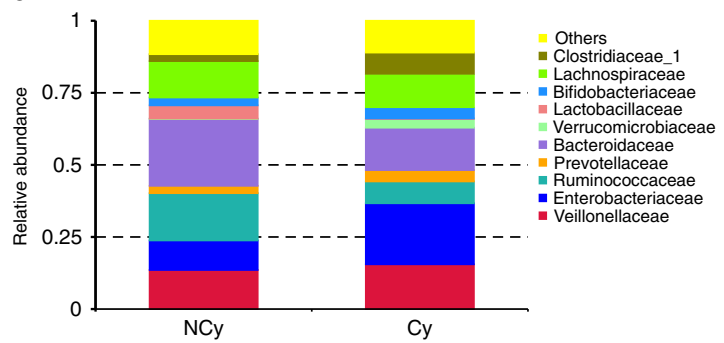

e

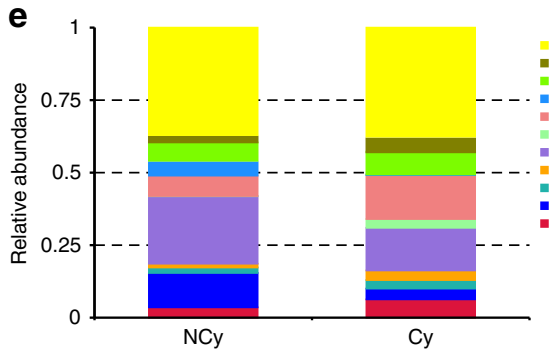

g

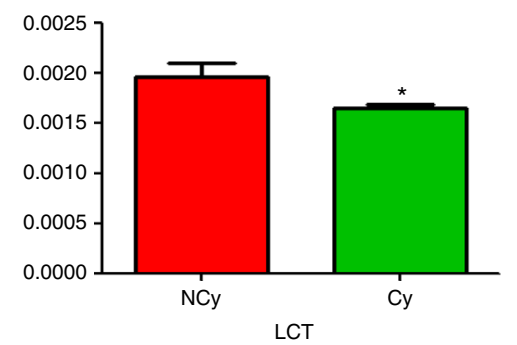

$\mathbf{h}_{35}$

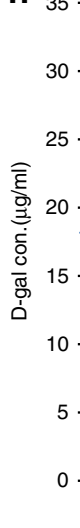

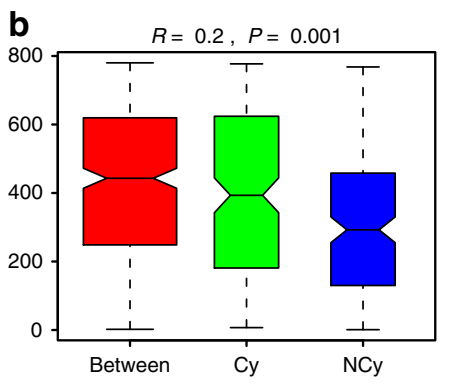

c

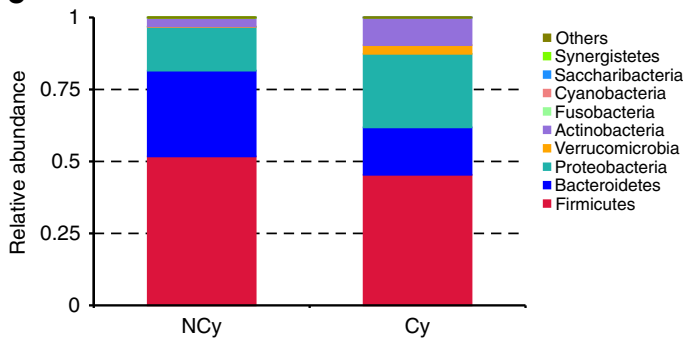

f $\mathrm{cy}$

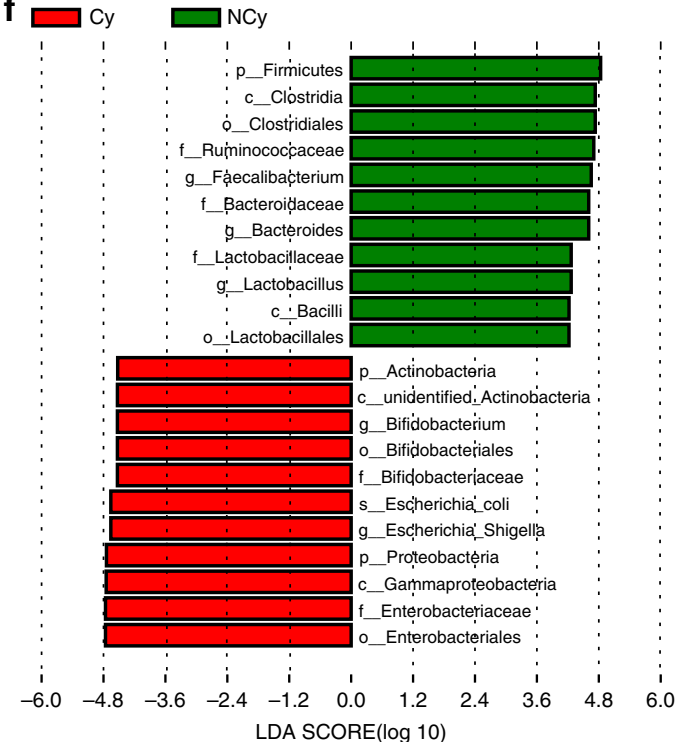

Others

Clostridium_sensu_stricto_1 Lactobacillus Escherichia-Shigella Akkermansia Klebsiella Prevotella_9 Faecalibacterium
LDA SCORE $(\log 10)$

LDA SCORE $(\log 10)$

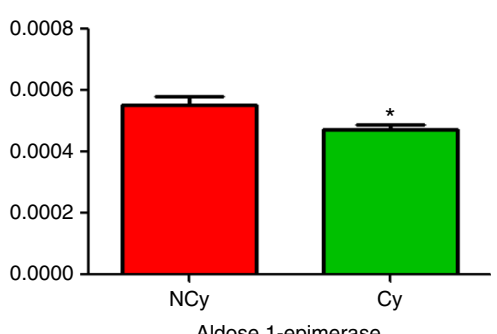

Aldose 1-epimerase

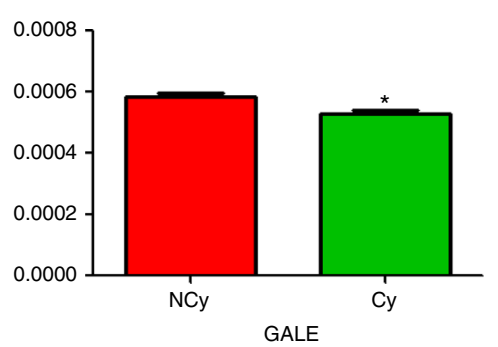

GALE

$$
R=-0.706
$$

$$
P<0.01
$$

Fig. 4 Profound hypoxia resulted in gut microbiota dysbiosis. a Rarefaction curves of the species number in the NCy and Cy groups ( $n=20$ per group). The curve in each group is nearly smooth when the sequencing data set is sufficiently large. $\mathbf{b}$ ANOSIM analysis of the beta diversity of the samples significantly separated the groups when $R>0$ and $P<0.05$. c-e The top 10 relative abundances of bacteria at phylum (c), family (d) and genus (e) levels in faecal samples from the NCy and Cy groups. $\mathbf{f}$ LEfSe analysis showed the association between two groups. $\mathbf{g}$ Metagenomic sequence analysis of the gene enrichment of enzymes that mediate D-galactose metabolism. LCT $(P=0.03)$, aldose 1-epimerase $(P=0.03)$, GALE $(P=0.01)$. h Pearson correlation analyses of Lactobacillus in stool and D-galactose in blood $\left({ }^{\star} P<0.05\right)$ 
negatively correlated with the D-galactose concentration in the patient's blood.

D-galactose accumulation and BMSC dysfunction in hypoxic rats. We established a chronic hypoxic rat model to confirm the effect of hypoxia on BMSCs. New-born Sprague-Dawley rats were housed in a hypoxic chamber under $10 \% \mathrm{O}_{2}$ or in ambient air for 3 weeks ${ }^{24}$. Haematocrit and haemoglobin levels in hypoxic rat blood were significantly higher than those in the normal group, and $\mathrm{pO}_{2}$ and $\mathrm{SO}_{2}$ levels were lower in the hypoxic group (Supplementary Fig. 5A), similar to the observations in patients with CCHD. The D-galactose concentration in rat blood (Fig. 5a), the Lactobacillus level in rat stool (Fig. 5b) and the expression of a variety of senescence markers (Fig. 5c) remained similar to those of the patients. D-galactose is primarily degraded by the liver, after being absorbed into the body via the intestine. We then tested $\mathrm{D}-$ galactose metabolism-related enzymes in rat liver. The expression of GALM/GALK2/GLB1/aldose reductase was unchanged, while GALK1 in rat livers decreased in the hypoxic group (Fig. 5d).

Lactobacillus supplementation rescues deficient BMSCs. The chronic hypoxic rats were intragastrically administered Lactobacillus $\left(1 \times 10^{9} \text { cells per day }\right)^{25}$ or saline for the last week. Stool samples from rats that received the Lactobacillus showed increased levels of Lactobacillus (Fig. 6a). In rats that were not supplemented with Lactobacillus, the peripheral D-galactose concentration was higher under hypoxic conditions (in the hypoxia/ - group) than under normal conditions $(\sim 2.88 \mu \mathrm{g} / \mathrm{ml}$ vs. $\sim 1.25 \mu \mathrm{g} / \mathrm{ml}$ in the normal/- group, $P<0.01$, one-way ANOVA followed by Tukey's test; Fig. 6b). When hypoxic rats were supplemented with Lactobacillus (hypoxia/+ group), the Dgalactose concentration was substantially reduced $(\sim 1.55 \mu \mathrm{g} / \mathrm{ml}$ vs. $\sim 2.88 \mu \mathrm{g} / \mathrm{ml}$ in the hypoxia/- group; $P<0.01$, one-way ANOVA followed by Tukey's test) and very similar to the levels detected in rats housed under normal oxygen conditions without Lactobacillus supplementation $(\sim 1.55 \mu \mathrm{g} / \mathrm{ml}$ vs. $\sim 1.25 \mu \mathrm{g} / \mathrm{ml}$ in the normal/- group, $P=0.083$, one-way ANOVA followed by Tukey's test).

Haematoxylin and eosin (H\&E) staining of the rat intestine revealed atrophy of the intestinal villus in the hypoxic group. Lactobacillus supplementation did not rescue this pathological change (Fig. 6c). The LCT activity in the rat intestine remained unchanged in all four groups $(P=0.936$, one-way ANOVA) (Fig. 6d). However, Lactobacillus supplementation of the rats rescued their BMSCs from senescence (Fig. 6e, g), poor cell proliferation (Fig. 6f), cell cycle arrest (Fig. 6h), multilineage differentiation deficiency (Supplementary Fig. 6 A-F) and reduction in the liver GALK1 levels (Supplementary Fig. 6G).

To investigate whether the supplement of Lactobacillus provide long-term beneficial effects, rats were divided randomly into three groups and housed in the hypoxic chamber for 2 weeks, then supplied with Lactobacillus or saline for 3 weeks (-/- group, supplied with saline for entire 3 weeks; +/-group, Lactobacillus 1 week followed by saline 2 weeks; $+/+$ group, supplied with Lactobacillus for entire 3 weeks). Compared to the -/-group (Supplementary Fig. 7A), the Lactobacillus level in rat stool was increased significantly in the $+/+$ group $(\sim 3.19$ fold, $P<0.01$, one-way ANOVA followed by Tukey's test), and no significant difference was observed between the $+/-$ and $-/-$ group $(P=$ 0.334 , one-way ANOVA followed by Tukey's test). The Dgalactose concentration (Supplementary Fig. 7B) in rat blood was a

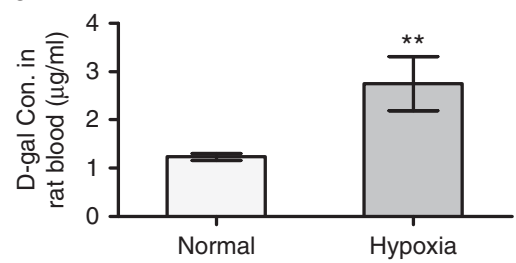

C
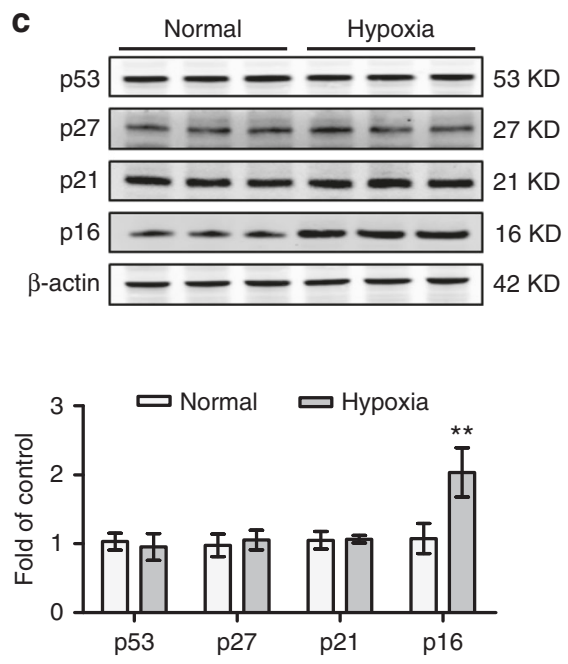

b

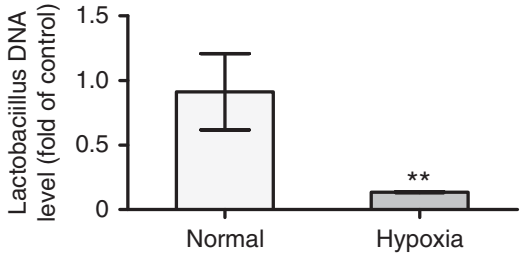

d
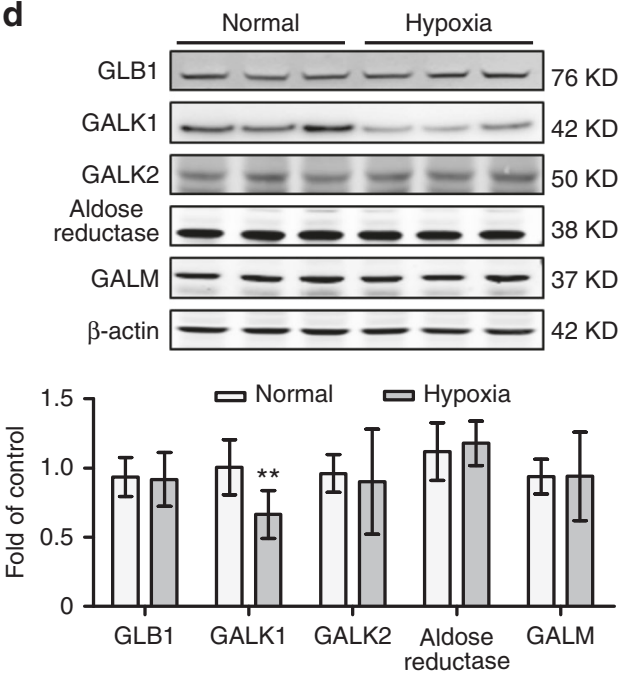

Fig. 5 Accumulation of D-galactose and BMSC dysfunction in a rat chronic hypoxia model. a New-born Sprague-Dawley rats were housed in normoxic or hypoxic chambers for 3 weeks, respectively ( $n=9$ per group). Peripheral blood samples were obtained, and the D-galactose concentrations were measured. b Stools from the rats described in a were subjected to qPCR to assess the Lactobacillus content. c BMSCs from a were cultured to passage 3 , and cell lysates were prepared to assess the levels of p53, p27, p21, p16 and $\beta$-actin by western blotting. $\mathbf{d}$ Western blotting was used to assess the protein levels of GALM, GALK1, GALK2, aldose reductase, GLB1 and $\beta$-actin in the rat liver. The data are the mean \pm SD from three independent experiments, and statistical significance was analysed using Student's $t$-test $\left({ }^{\star \star} P<0.01\right)$ 
lower in the $+/+$ group $(\sim 1.38 \mu \mathrm{g} / \mathrm{ml}$ vs. $\sim 3.40 \mu \mathrm{g} / \mathrm{ml}$ in $-/-$ group, $P<0.01$, one-way ANOVA followed by Tukey's test) and back to higher level in the $+/-$ group $(\sim 3.00 \mu \mathrm{g} / \mathrm{ml}$ vs. $\sim 3.40 \mu \mathrm{g} /$ $\mathrm{ml}$ in $-/-$ group, $P=0.164$, one-way ANOVA followed by
Tukey's test). The rescued BMSCs (as described above) showed a premature senescence phenotype in the $+/-$ group, while continuous supplementation with Lactobacillus $(+/+$ group) show decreased proportion in SA- $\beta$-galactosidase $(\sim 20.31 \%$ in the

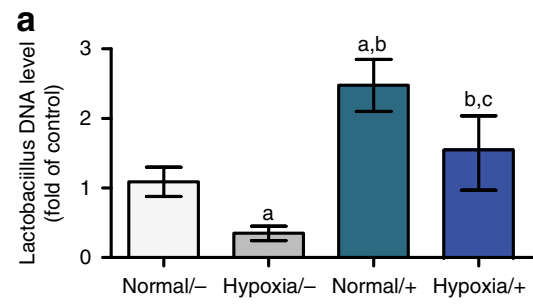

b

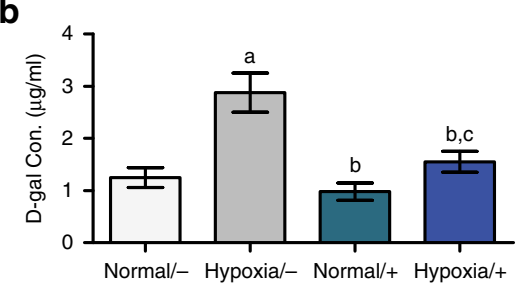

d

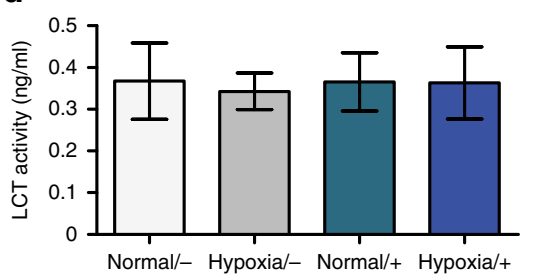

f
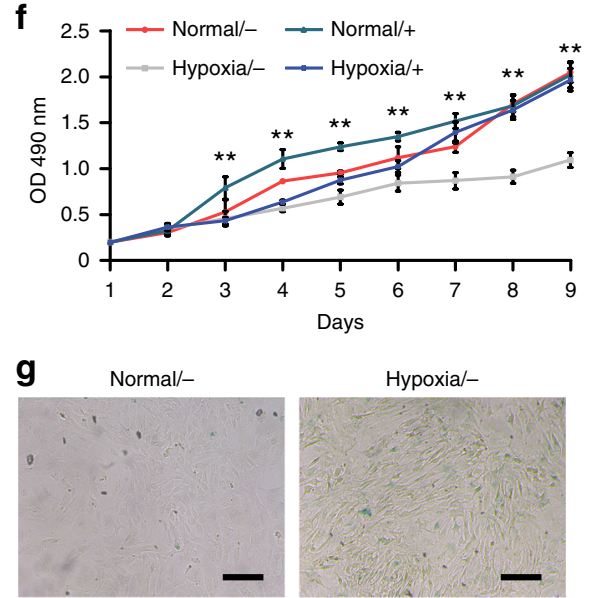

Normal/+
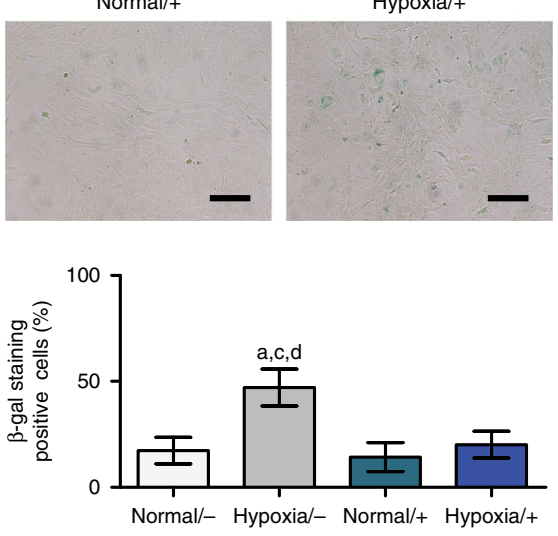

C

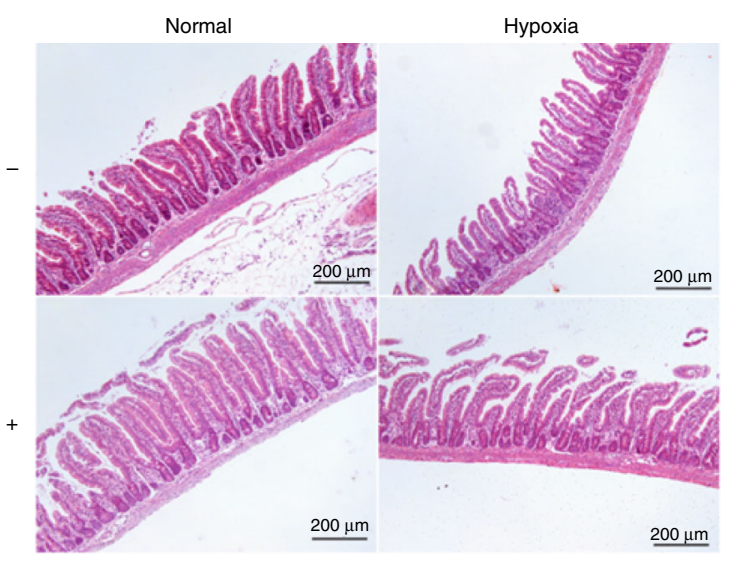

e
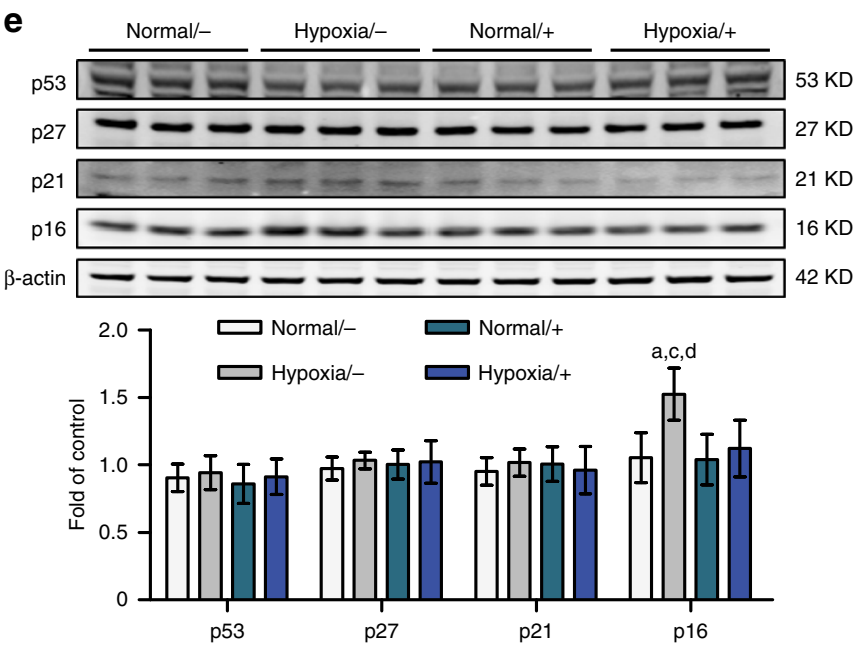

h
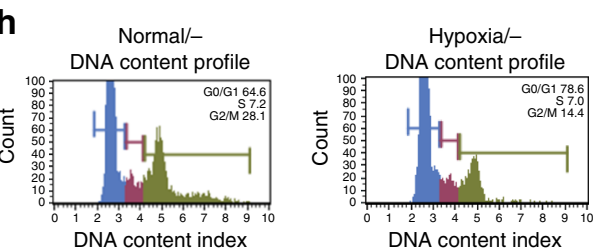

Normal/+
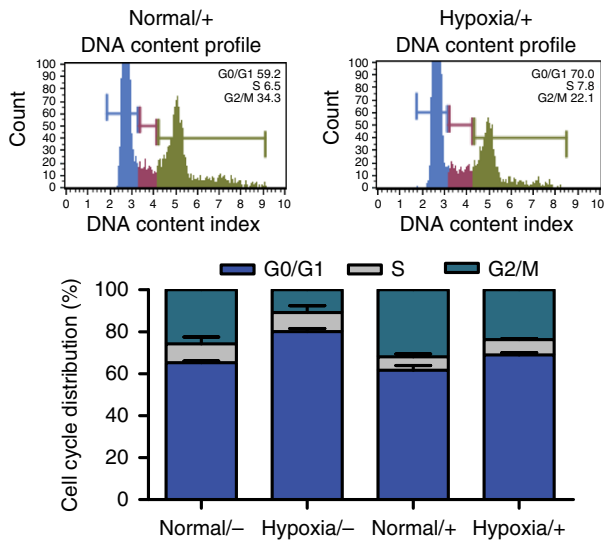
$+/+$ group vs. $\sim 43.00 \%$ in the $+/-$ group, $P=0.013$, one-way ANOVA followed by Tukey's test; Supplementary Fig. 7C).

\section{Discussion}

In the present study, we showed first that the chronic profound hypoxia experienced by patients with CCHD adversely influences BMSCs. Chronic profound hypoxia results in an altered diversity of gut microbial communities with a marked decrease in Lactobacillus and reduction of enzymes that degrade D-galactose. Cumulative D-galactose in the blood and BM induced BMSC premature senescence through ROS accumulation. Moreover, Lactobacillus supplementation in a rat model could recover the function of deficient BMSCs in the hypoxic rat model (Fig. 7).

Our results showed reduced oxygen tension in the BM of patients with CCHD than in NCy children. Low oxygen tension is essential for maintaining the pluripotency of stem cells ${ }^{17}$, and the proliferation of BMSCs is enhanced when they are cultured in vitro under low $\mathrm{pO}_{2}$ conditions $\left(2-3 \% \mathrm{O}_{2}\right)^{26}$. However, our results imply that the hypoxia-mediated protection of stemness is jeopardized in patients with CCHD. Previous studies have shown that various metabolites of cellular metabolism can affect BMSCs. It has been reported that 2,5-hexanedione can induce rat BMSC apoptosis ${ }^{27}$ and that glyoxal can induce senescence in human $\mathrm{BMSCs}^{28}$. We explored metabolomics to elucidate distinctive BM metabolism signatures. Although no changes in 2,5-hexanedione and glyoxal were observed, we found that hypoxia in patients with CCHD altered the energy supply in BM and led to an accumulation of D-galactose.

As a constituent of milk, lactose is the major nutrient for infants and young children. Lactose is degraded to glucose and Dgalactose in the intestine and absorbed into the body. Most of the absorbed D-galactose is degraded in the liver for energy production, while little is used for early development in humans ${ }^{29}$. Dgalactose flows through the arteries to penetrate the cortical bone and medullary canal and then into the BM metaphyseal region and arterial capillaries. Based on the specified physiological structure of the $\mathrm{BM}$, the peripheral blood and $\mathrm{BM}$ are well established for their interaction. Several studies have demonstrated that the metabolite distribution is significantly different but correlated between BM and peripheral blood ${ }^{30,31}$. Our results showed that the D-galactose concentration was significantly increased in patients with CCHD, in both peripheral blood and BM. A series of enzymes that metabolize D-galactose are also present in BMSCs, but there were no significant differences between the NCy and Cy groups (Supplementary Fig. 4B). Dgalactose has been extensively leveraged in mouse models of ageing ${ }^{32,33}$. Therefore, the accumulated excessive D-galactose in BM could exert a negative effect on BMSCs.

High-dose D-galactose can induce premature ageing of astrocytes, foetal fibroblasts and neuroblasts 32,34 . A number of reports have suggested that the ageing model induced by D-galactose is associated with oxidative stress ${ }^{33}$. Subsequent studies have demonstrated that ROS accumulation mainly accounts for this process $^{23}$. Strikingly, although the D-galactose concentration adopted in our in vitro cell culture study was far less than that used in mouse models of ageing, it still induced premature senescence of BMSCs under hypoxic conditions. Moreover, an increased level of ROS was detected only in BMSCs from patients with CCHD as well as the Hypo-H-Gal group. Therefore, profound hypoxia might be conducive to the function of D-galactose in downstream processes via alterations in energy production and ROS accumulation.

Intriguingly, BMSCs from patients with CCHD exhibited much worse antiapoptotic capability than those from $\mathrm{NCy}$ patients, indicative of the propensity for apoptosis in CCHD patients. Indeed, D-galactose has been reported to harbour a cytotoxic effect on cells, either in expediting senescence or in inducing apoptosi ${ }^{35}$. It is worth noting that both cytotoxic effects largely depend on ROS via the oxidative metabolism of D-galactose as well as advanced glycation end products ${ }^{36}$. It has been proposed that cells enter a state of senescence when the ROS level is sublethal ${ }^{37}$ or apoptosis after exposure to high levels of ROS ${ }^{38}$. Although complete comprehension of the molecular mechanisms involved in cellular senescence and apoptosis remained to be attained, it is believed that the ROS level plays a pivotal role in balancing the two physiological processes $^{39}$. Moreover, despite some controversies, ageing may predispose related cells to apoptosis by enhancing mitochondrial dysfunction $^{40,41}$. The mechanism responsible for this process clearly merits further characterization.

The gut microbiota had been reported extensively to be involved in lactose and D-galactose metabolism since the Dgalactose metabolism pathway (Leloir pathway) is highly conserved and the enzymes in the Leloir pathway are also encoded by genes in Lactobacillus ${ }^{42}$. Lactose digestion can be improved by the modulation of colonic bacteria ${ }^{43}$. As human gut symbionts, the diversity of microbiota can, in general, be largely influenced by the diverse physiological states of the host ${ }^{44}$. We found that the Firmicutes/Bacteroides ratio increased in the Cy group, which is similar to findings obtained for patients with hypertension and obesity $^{19}$. Enterobacteriaceae increased in the Cy group, whereas Lactobacillus, Lachnospiraceae, Ruminococcaceae and Faecalibacterium decreased. Previous studies have shown that many members of the Enterobacteriaceae family are pathogens, such as Shigella, one of the leading bacterial causes of diarrhoea ${ }^{45}, 46$. Lachnospiraceae may protect against colon cancer in humans by producing butyric acid ${ }^{47}$. Additionally, Faecalibacterium shows greater depletion in the gut microbiota of older heart failure patients ${ }^{48}$. In the present study, we noted that Lactobacillus markedly decreased in both patients with CCHD and in hypoxic rats. Intermittent hypoxia has been reported to alter the gut microbiota diversity in a mouse model of sleep apnoea, in which Lactobacillus does not appear to significantly change ${ }^{49}$. However, it has been noted that differing hypoxic status, whether intermittent or chronically profound, should be taken into account for commensal bacteria modulation. The gut microbiota is known to impact host distal organs via bioactive metabolites ${ }^{19}$. We found a strong negative correction between Lactobacillus reduction in the gut microbiota and D-galactose accumulation in the peripheral blood. Metagenomics sequencing of stool samples from children

\footnotetext{
Fig. 6 Functional recovery of deficient BMSCs through Lactobacillus supplementation in the rat hypoxia model. a Lactobacillus casei CRL431 (+) or saline ( - ) was administered intragastrically to rats housed in normoxic or hypoxic chambers ( $n=9$ per group). DNA was extracted from stool samples and subjected to real-time QPCR analysis to assess the levels of Lactobacillus DNA. $\mathbf{b}$ The D-galactose concentration in peripheral blood samples was detected in the rats described in a. c Rat intestines were subjected to H\&E staining. $\mathbf{d}$ Intestines from the rats described in a were subjected to ELISA of LCT activity. e-h BMSCs from rats described in a were isolated and cultured to passage 3. Cell lysates were analysed for ageing-related proteins by western blotting (e). Cells were subjected to MTT assays (f), SA- $\beta$-gal analysis (scale bar, $100 \mu \mathrm{m}$ ) (g) and FACS analysis (h) to assess cell growth, premature senescence status and cell cycle distribution, respectively. Data represent the means \pm SD from three independent experiments, and statistical significance was analysed by one-way ANOVA followed by Tukey-Kramer multiple comparisons. ${ }^{a} p<0.05$ compared with normal - . ${ }^{b} p<0.05$ compared with hypoxia/-. ${ }^{\mathrm{c}} P<0.05$ compared with normal/ $+.{ }^{\mathrm{d}} P<0.05$ compared with hypoxia/ $\left.+{ }^{\star \star} P<0.01\right)$
} 


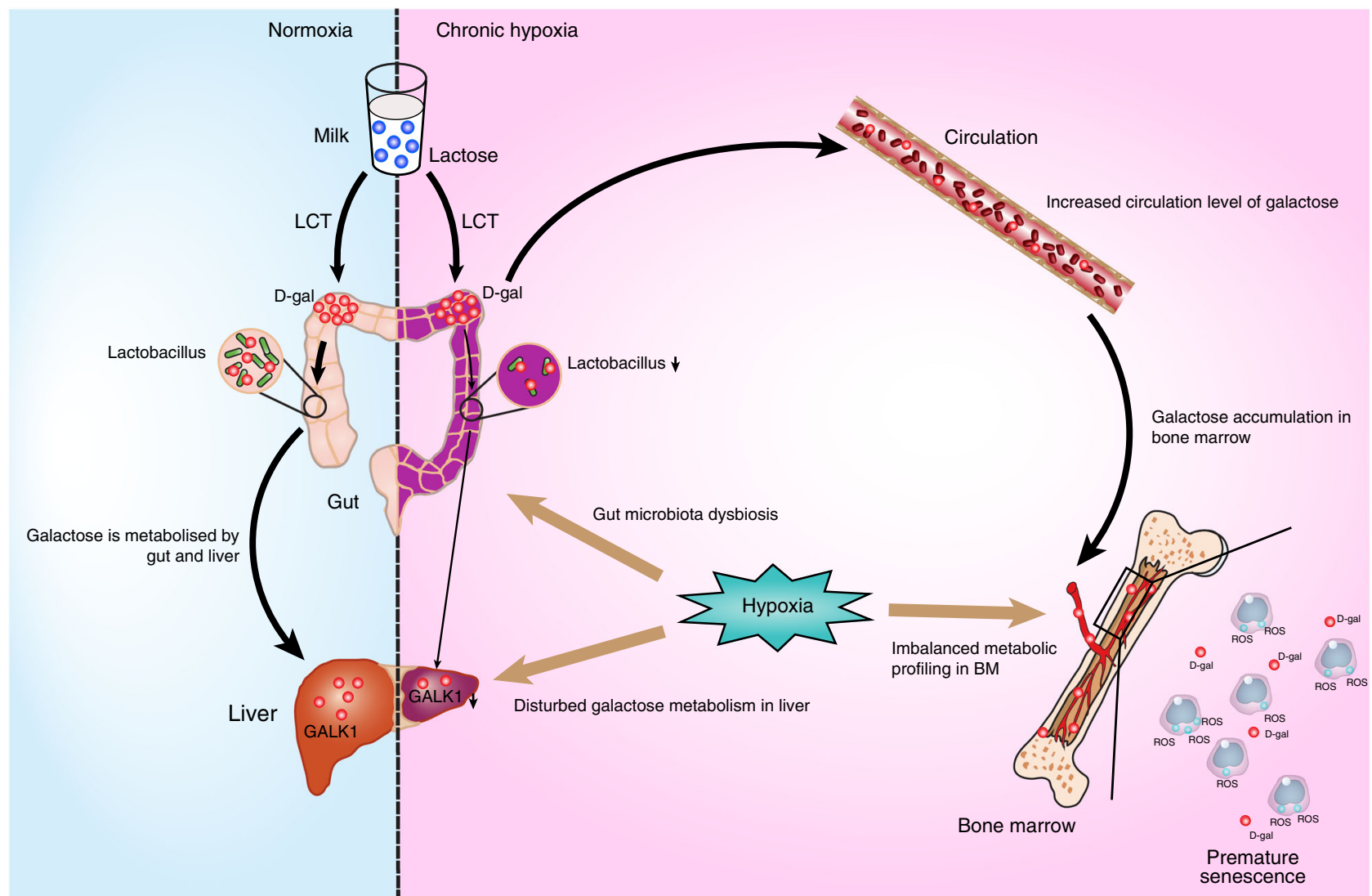

Fig. 7 Summary Figure. As a constituent of milk, lactose is the major nutrient for infants and young children. Chronic profound hypoxia induced by CCHD results in alterations in the diversity of gut microbial communities, with markedly decreased Lactobacillus and a reduction in enzymes that degrade $D$ galactose. Cumulative D-galactose in the blood and BM induced premature senescence of BMSCs through ROS accumulation

revealed a distinct loss of D-galactose metabolism-related gene enrichment in the Cy group. These finding strongly indicated that the accumulation of D-galactose in the blood and BM could result from gut microbiota-derived metabolic alterations.

Lactose is also degraded by LCT, which is secreted by the intestinal gland around the brush border area. We did not observe impaired LCT activity in the intestine in the rat hypoxia model. Likewise, intestinal mucosa atrophy was noted in our model, which has been previously observed both in hypoxia rat ${ }^{50}$ and patients with chronic obstructive pulmonary disease ${ }^{51}$, indicating impaired mucosal barrier fortification and thus the entry of more bacterial metabolites into the circulation ${ }^{50}$. Given this case, we cannot exclude the fact that gut dysgenesis independent of microbiome is also a cause for the observed phenotype. We found that the replenishment of hypoxic rats with Lactobacillus could fully restore the deficient BMSCs, while the intestinal mucosa atrophy did not fully recover even after probiotic therapy. Therefore, the alterations of the gut microbial communities might be the main reason for the deficient BMSCs, and complete surgical repair of cardiac anomalies is warranted to relieve hypoxia and restore the integrity of the mucosal barrier.

Lactobacillus is one of the most important probiotic bacteria responsible for lactose and D-galactose metabolism in the intestinal tract. Lactobacillus is well used in microbial-mediated therapy. Although the atrophied intestinal villus with impaired mucosal barrier fortification and reduced GALK1 expression in the liver were observed in the rat hypoxia model, we found that the replenishment of hypoxic rats with Lactobacillus could reduce the accumulation of D-galactose and restore the deficient BMSCs. As a simple preventive approach, probiotics could produce antimicrobial substances, prevent pathogen and toxin adsorption, and modulate the immune response $\mathrm{e}^{52}$, which has been applied in some clinical studies to prevent necrotizing enterocolitis among the neonatal and paediatric population ${ }^{22}$. In the clinical setting, oral probiotic supplementation is more convenient than faecal microbiota transplantation, especially for children with CCHD. However, when Lactobacillus was removed from the diet of hypoxic rats, the BMSCs showed premature senescence again. It strongly suggested that continuous supplementation was necessary before the correction of hypoxia status.

Undoubtedly, our study has some limitations. First, various diseases are known to be involved in systematic chronic hypoxia. The disease model for this study confined merely to CCHD patients obviously appears to be incomplete. Second, the patients enrolled in the present study were young children. A recent study showed a link between Lactobacillus and blood pressure among healthy adults $^{53}$. The effect of hypoxia and gut microbiota remains to be further characterized in adults. Third, given that BMSCs and haematopoietic stem cells (HSCs) share the same microenvironment, it is conceivable that the accumulated D-galactose in CCHD patients likely influenced the function of HSCs and even other progenitor cells residing in the bone marrow. Recently, Randa et al. ${ }^{54}$ has suggested that the increased immature platelet fraction in CCHD patients may denote peripheral platelet destruction. Apparently, haematopoiesis is perturbed to some extent in CCHD patients. However, the effect of hypoxia driven by CCHD on HSCs remains far to be determined owing to the lack of related studies. The dynamic change in HSCs and likely malfunction of haematopoiesis in CCHD patients requires further study.

In summary, BMSCs from patients with CCHD were predisposed to premature senescence, which might have resulted from gut dysbiosis and gut microbiota-derived D-galactose 
accumulation. Lactobacillus supplementation restored the deficient BMSCs. Our findings suggested that oral Lactobacillus supplementation might enhance the efficiency of autologous BMSC-based regenerative therapy and open possibilities to improve stem cell-based cures for patients with CCHD.

\section{Methods}

Study approval. This study was approved by the ethics committee of Fuwai Hospital, and the study protocol conformed to the ethical guidelines of the 1975 Declaration of Helsinki. The parents of all enrolled patients provided informed consent.

All animal procedures were conducted in accordance with the procedures reviewed and approved by the Care of Experimental Animals Committee. All efforts were made to minimize animal suffering.

Patients. Patients undergoing surgical repair for CHD were intubated and supplemented with $21 \%$ oxygen after induction of general anaesthesia in the operating room. Next, 1-2 $\mathrm{ml}$ of BM was aspirated from the sternum of the 90 patients undergoing open-chest cardiac surgery. The samples were used for metabolic profiling, cell culture and phenotype and blood gas analyses as described.

Rat. A rat model was established to mimic the chronic hypoxic status experienced by patients with $\mathrm{CCHD}^{24}$. Briefly, new-born Sprague-Dawley rats were divided randomly into normal and hypoxic groups. The hypoxic group of rats were housed in a hypoxic chamber (ProOx110, Biospherix, NY) under $10 \% \mathrm{O}_{2}$ for 3 weeks, whereas rats in the normal group were housed in ambient air for the same period.

For supplementation with Lactobacillus, new-born Sprague-Dawley male rats were divided randomly into four groups ( $n=9$ per group); two groups were housed in a normal environment, while the other two were housed in a $10 \% \mathrm{O}_{2}$ chamber for 3 weeks. One group in the normoxic chamber (normal/+ group) and one group in the hypoxic chamber (hypoxia/+ group) were intragastrically administered Lactobacillus casei CRL431 (Chr. Hansen A/S, Horsholm) for the last week $\left(1 \times 10^{9} \text { cells per day }\right)^{25}$, while the other groups in the normoxic (normal/group) and hypoxic (hypoxia/ - group) chambers received saline intragastrically. Rat blood, stool, intestine and BMSCs were obtained and analysed.

To check whether the Lactobacillus effects on hypoxic rats are permanent or simply acute, new-born Sprague-Dawley male rats were divided randomly into three groups ( $n=6$ per group) and housed in the hypoxic chamber. Two weeks later, two of them were supplied with Lactobacillus $(+/+$ group and $+/-$ group) for 1 week, while the $-/-$ group was supplied with saline $(-/-)$. Then the $+/+$ group was supplied with Lactobacillus for additional 2 weeks, and the rat in the $+/-$ and $-/$ - groups received saline instead. Rat blood, stool and BMSCs were obtained and analysed.

Cell culture and phenotype analysis. Human and rat BMSCs were isolated and cultured in Dulbecco's Modified Eagle's Medium (DMEM, HyClone) supplemented with $10 \%$ foetal bovine serum (FBS) and antibiotics at $37^{\circ} \mathrm{C}$ in $5 \% \mathrm{CO}_{2}$. The cell morphology was observed with an inverted microscope. Cell surface markers were measured with the Human MSC Analysis Kit (BD, Biosciences, San Jose, CA) by following the manufacturer's instructions. Third-passage BMSCs were cultured. CD90, CD105, CD73, CD44, CD34, CD11b, CD19, CD45 and HLA-DR were all tested by flow cytometry for the phenotypic analysis.

To evaluate the effect of D-galactose on BMSCs, BMSCs from children in the $\mathrm{NCy}$ group were cultured in an incubator with low $(4 \%)$ or normal $(21 \%) \mathrm{O}_{2}$, and the cells were exposed to normal $(50 \mu \mathrm{g} / \mathrm{ml})$ or high $(90 \mu \mathrm{g} / \mathrm{ml})$ concentrations of Dgalactose (Sigma, St. Louis, MO). These cells were designated Hypo-N-Gal, Hypo$\mathrm{H}-\mathrm{Gal}$, Nor-N-Gal and Nor-H-Gal, respectively. After 21 days, the BMSCs were harvested for $\beta$-galactosidase staining and the MTT and cell cycle assays.

Antibodies and western blot analysis. Cell lysate was extracted by RIPA buffer with protease inhibitors (Roche). Protein samples were run on sodium dodecyl sulfate-polyacrylamide gel and transferred onto transferred onto nitrocellulose membranes by an iBlot 2 dry blotting system (Thermo Fisher Scientific). Membranes were blocked in $5 \%$ non-fat dry milk in TBST ( $100 \mathrm{mmol} / \mathrm{l}$ Tris, $\mathrm{pH} 7.5,0.9 \% \mathrm{NaCl}$, $0.1 \%$ Tween-20) for $1 \mathrm{~h}$ and incubated with primary antibodies overnight at $4{ }^{\circ} \mathrm{C}$. Membranes were washed with TBST 3 times and incubated for $1 \mathrm{~h}$ with Dylight 800 goat anti-mouse-IgG (1:2000, EarthOx, E032810) or Dylight 800 goat anti-rabbit-IgG secondary antibody (1:2000, EarthOx, E032820) at room temperature. Signals were detected by using Odyssey CLx Western Blot Detection System.

The senescence-related gene expression levels in passage-3 BMSCs were analysed. Monoclonal anti-P16 (1:1000, Abcam, Cat\# ab108349), anti-P53 (1:1000, Abcam, Cat\# ab179477), anti-P27 (1:1000, Abcam, Cat\# ab32034), anti-P21 (1:1000, Abcam, Cat\# ab109520) and anti- $\beta$-actin (1:10000, Proteintech, Cat\# 60008-1-Ig) were used.

D-gal metabolism-related enzymes were analysed in human BMSCs, rat BMSCs and rat liver. Monoclonal anti-GLB1 (1:50000, Abcam, Cat\# ab128993), anti-aldose reductase (1:100, Santa Cruz, Cat\# sc-373953) and anti-GLAM (1:100, Santa Cruz, Cat\# sc-166304) and polyclonal anti-GALK1 (1:1000, abcam, Cat\# ab65123) and anti-GALK2 (1:1000, abcam, Cat\# ab153815) were used.
The uncropped western blots and all the western blot repeats (at least three replicates) were included in Supplementary Figure 8 and Supplementary Figure 9 , respectively.

$\boldsymbol{\beta}$-Galactosidase staining. BMSCs were cultured to passage 3 and plated in 6-wel plates for $\beta$-galactosidase staining following the manufacturer's instructions provided with the $\beta$-Galactosidase Staining Kit (Cell Signaling Technology, Danvers, $\mathrm{MA})$. Cells were washed in phosphate-buffered saline (PBS) and fixed for $5 \mathrm{~min}$ at room temperature in $4 \%$ formaldehyde. Then cells were incubated overnight (without $\mathrm{CO}_{2}$ ) at $37^{\circ} \mathrm{C}$ with SA- $\beta$-gal stain solution $(1 \mathrm{mg} / \mathrm{ml} \mathrm{X}$-gal, $40 \mathrm{mM}$ citric acid/sodium phosphate, $\mathrm{pH} 6.0,5 \mathrm{mM}$ potassium ferrocyanide, $5 \mathrm{mM}$ potassium ferricyanide, $150 \mathrm{mM} \mathrm{NaCl}, 2 \mathrm{mM} \mathrm{MgCl} 2$ ). Images were collected using a DM750 microscope (Leica, Germany). Twenty fields of each section were randomly selected to calculate the ratio of $\beta$-galactosidase-positive cells

MTT assays. Cells were plated in 96-well plates at a density of $2.0 \times 10^{3}$ cells per well for the MTT assay. The optical density at $490 \mathrm{~nm}$ was determined for each well using an absorbance plate reader from the first day to the eighth day, and the growth curves were plotted to estimate the proliferation potential of BMSCs from various groups.

Cell cycle assays. For the cell cycle assay, BMSCs were plated in 6-well plates and treated with serum-free medium for $12 \mathrm{~h}$ to achieve cell synchronization. Next, DMEM supplemented with $10 \%$ FBS was added to promote BMSC division. After $24 \mathrm{~h}$, BMSCs were collected, incubated in $37{ }^{\circ} \mathrm{C}$ with RNase A $(20 \mu \mathrm{g} / \mathrm{ml}$; Thermo) for $30 \mathrm{~min}$ and then stained with propidium iodide (PI; $50 \mu \mathrm{g} / \mathrm{ml}$ ) (Beyotime, China). Cell cycle analysis was performed using BD accuri C6.

Antiapoptosis assays. BMSCs were cultured with serum-free DMEM in a hypoxia incubator for $12 \mathrm{~h}$ to induce cell apoptosis as previously described ${ }^{55}$. The cells were then collected and incubated with the fluorescent dye Annexin V and PI. Apoptosis was measured by flow cytometry with a BD accuri C6.

Multilineage differentiation potential assay of BMSCs. For osteogenic differentiation, BMSCs were seeded at $2 \times 10^{4} / \mathrm{cm}$ in 6 -well plates. In all, $0.1 \mathrm{mM} \mathrm{dex}-$ amethasone, $10 \mathrm{mM} \beta$-glycerol phosphate and $50 \mathrm{mM}$ ascorbate were added to the medium to induce differentiation ${ }^{56}$. The medium was changed every 3 days. Seven days later, ALP activity were measured by the Alkaline Phosphatase Detection Kit (Sigma) according to the manufacturer's instructions. And 3 weeks later, cells were fixed in $4 \%$ paraformaldehyde for $15 \mathrm{~min}$, washed 3 times with PBS and then stained with Alizarin red $(1 \%$ in $0.1 \mathrm{M}$ Tris- $\mathrm{HCl}(\mathrm{pH}=8.3)$ ) for $5 \mathrm{~min}$ to assess the degree of osteogenic differentiation,

BMSCs were seeded at $2 \times 10^{4} / \mathrm{cm}$ in 6-well plates. Adipogenic differentiation was induced in the medium with $1 \mu \mathrm{M}$ dexamethasone, $200 \mu \mathrm{M}$ indomethacin, 0.5 $\mu \mathrm{M}$ 1-methyl-3-isobutylxanthine and $10 \mu \mathrm{g} / \mathrm{ml}$ insulin ${ }^{57}$. Three weeks later, cells were fixed in $4 \%$ paraformaldehyde for $15 \mathrm{~min}$, washed 3 times with PBS, then stained with $(3 \mathrm{~g} / \mathrm{l})$ Oil Red O for $30 \mathrm{~min}$, washed in $75 \%$ ethanol and stained with haematoxylin for $1 \mathrm{~min}$ to assess adipogenic differentiation by microscope DM750 (Leica, Germany). Twenty fields of each section were randomly selected to calculate the ratio of adipogenic differentiation.

To induce chondrogenic differentiation, BMSCs were gently centrifuged $(250 \times$ $\mathrm{g}, 10 \mathrm{~min}$ ) to form a pelleted micro-mass and cultured with $0.1 \mu \mathrm{M}$ dexamethasone, $0.17 \mathrm{mM}$ ascorbate, $10 \mu \mathrm{g} / \mathrm{l} \mathrm{TGF}-\beta 3$, ITS + supplement, $1 \mathrm{mM}$ sodium pyruvate and $0.35 \mathrm{mM}$ proline ${ }^{57}$. Three weeks later, cell pellets were fixed in $4 \%$ paraformaldehyde for $30 \mathrm{~min}$ and dehydrated through an alcohol gradient series, embedded in paraffin wax blocks. Before staining, 2- $\mu \mathrm{m}$-thick sections were dewaxed in xylene, rehydrated through decreasing concentrations of ethanol, blocked in 5\% goat serum (with $0.3 \%$ Triton X-100) for $1 \mathrm{~h}$ at $37^{\circ} \mathrm{C}$ and incubated with Collagenase II antibody (1:250, Santa Cruz, Cat\# sc-52658) overnight at $4{ }^{\circ} \mathrm{C}$. Washed 3 times with PBS and incubated with FITC-labelled goat anti-mouse-IgG (1:1000, ZSGB-Bio, Cat\# ZDR5210) at room temperature for $1 \mathrm{~h}$. Images were collected using a DM750 microscope (Leica, Germany). Twenty fields of each section were randomly selected to calculate the ratio of chondrogenic differentiation.

All of the reagents used in multilineage differentiation assay are from Sigma.

Metabolic profiling analysis. BM supernatants were collected from the patients after centrifugation. Next, $350 \mu \mathrm{l}$ methanol was added to $100 \mu \mathrm{l}$ BM supernatants containing $50 \mu \mathrm{l}$ of L-2-chlorophenylalanine as an internal standard. The lyophilized metabolite extract was then sequentially oximated with methoxyamine hydrochloride and silylated with BSTFA reagent (1\% TMCS, v/v; REGIS Technologies, USA). Metabolomic analysis was performed using an Agilent 7890 gas chromatograph system coupled to a Pegasus HT time-of-flight mass spectrometer (GC-TOF/MS, LECO, St. Joseph, MI, USA).

Chroma TOF4.3× software (LECO, St. Joseph, MI, USA) was used for raw data analysis, including raw peak extraction, baseline data filtering, calibration of the baseline, peak alignment, deconvolution analysis, peak identification and integration of the peak area. The SIMCA-P 13.0 software package (Umetrics, Umea, Sweden) was then used for principal component analysis (PCA), PLS-DA and OPLS-DA. A leave- 
one-out validation was used to estimate the robustness and predictive ability of the mode. Metabolites that were significantly different between the $\mathrm{NCy}$ and $\mathrm{Cy}$ groups were screened using the OPLS-DA model. The principal component of the VIP $>1.0$ was first selected as a changed metabolite. The nonparametric univariate method (Mann-Whitney-Wilcoxon test) was used to analyse metabolites that differed in abundance between the NCy group and the Cy group corrected for the FDR to ensure that the peak of each metabolite was reproducibly detected in the samples. The metabolites detected using GC-TOF/MS were identified by searching against the LECO/Fiehn Metabolomics Library and validated with standard substances if available. In addition, the Kyoto Encyclopaedia of Genes and Genomes (http://www. genome.jp/kegg/) and MetaboAnalyst 3.0 (http://www.metaboanalyst.ca/ MetaboAnalyst/) were utilized to analyse the metabolic pathways.

\section{Analysis of oxygen concentration and D-galactose in blood and BM. Blood gas} analysis for the freshly harvested BM was performed according to a previously described protocol ${ }^{58}$. Briefly, $1-2 \mathrm{ml}$ of whole $\mathrm{BM}$ marrow or blood was aspirated into a blood gas syringe. The $\mathrm{PO}_{2}$ and $\mathrm{SO}_{2}$ were measured by blood gas analyser CCX1 (NOVA, USA).

Blood samples $(1-2 \mathrm{ml})$ were obtained and allowed to stand for $30 \mathrm{~min}$, centrifuged at $1000 \times g$ for $10 \mathrm{~min}$ at room temperature and then transferred the supernatant to liquid nitrogen immediately. All samples were stored at $-80^{\circ} \mathrm{C}$ until use.

A Galactose Colorimetric/Fluorometric Assay Kit (Sigma, St. Louis, MO) was used to measure D-galactose in the BM supernatant and peripheral blood according to the manufacturer's instructions in both the human and rat. In brief, $50 \mu \mathrm{l}$ serum were incubated with $50 \mu \mathrm{l}$ Master Reaction Mix ( $44 \mu \mathrm{l}$ Galactose Assay Buffer, $2 \mu \mathrm{l}$ Galactose Probe, $2 \mu$ l Galactose Enzyme Mix, $2 \mu \mathrm{lHRP}$ ) for $30 \mathrm{~min}$ at $37^{\circ} \mathrm{C}$ in dark. The absorbance at $570 \mathrm{~nm}$ were measured.

\section{Stool 165 ribosomal RNA sequencing and metagenomics sequencing. Forty} patients were randomly chosen for stool $16 \mathrm{~S}$ ribosomal RNA and metagenomics sequencing ( $n=20$ per group). Fresh faecal samples were collected before cardiac surgery. The inside portions of the stool were frozen in liquid nitrogen within $1 \mathrm{~h}$ of collection. All samples were stored at $-80^{\circ} \mathrm{C}$ until use.

Bacterial genomic DNA from stools was isolated using a QIAamp DNA Stool Mini Kit (QIAGEN). The 16S ribosomal RNA sequencing and metagenomics sequencing were used to analyse differences between the $\mathrm{NCy}$ and Cy groups on the Illumina platform at Novogene Bioinformatics Technology Co., Ltd. The 16S rRNA genes of distinct regions (V3-V4) were amplified using specific primers (341F: CCTAYGGGRBGCASCAG; 806R: GGACTACNNGGGTATCTAAT) Sequencing libraries were generated using the TruSeq ${ }^{\circledR}$ DNA PCR-Free Sample Preparation Kit (Illumina, USA). The library was sequenced on an Illumina HiSeq2500 platform, and 250-bp paired-end reads were generated. Sequence analyses were performed using the Uparse software. Sequences with $\geq 97 \%$ similarity were assigned to the same operational taxonomic units (OTUs). Additionally, based on the normalized OTUs, alpha diversity was applied to analyse the complexity of species diversity for the samples. ANOISM analysis was used to evaluate differences among the samples in species complexity.

For metagenomics sequencing, a total of $1 \mu \mathrm{g}$ of DNA per sample was used as input material for the DNA sample preparations. Sequencing libraries were generated using the NEBNext ${ }^{\circledR}$ Ultra ${ }^{\text {ma }}$ DNA Library Prep Kit for Illumina (NEB, USA) according to the manufacturer's recommendations, and index codes were added to attribute the sequences to each sample. Briefly, the DNA sample was fragmented by sonication to a size of $350 \mathrm{bp}$, and then the DNA fragments were end-polished, Atailed and ligated with the full-length adaptor for Illumina sequencing for further PCR amplification. Finally, the PCR products were purified (AMPure XP system), and the libraries were analysed for size distribution using an Agilent2100 Bioanalyser and quantified using real-time PCR. Clustering of the index-coded samples was performed on a cBot Cluster Generation System according to the manufacturer's instructions. After cluster generation, the library preparations were sequenced on an Illumina HiSeq platform, and paired-end reads were generated.

Real-time PCR was performed to identify changes in the bacterium in rat and human stool. The following primer pairs were used: Lactobacillus-1 F: 5'-AGCA GTAGGGAATCTTCCA-3', Lactobacillus-1 R: 5'-CACCGCTACACATGGAG-3', Lactobacillus-2 F: 5'-GCCTTGTACACACCGCCCGT-3', Lactobacillus-2 R: 5'CTCAAAACTAAACAAAGTTTC-3', Universal bacterial primer F: 5'-CCTACG GGAGGCAGCAG-3', and Universal bacterial primer R: 5'-ATTACCGCGGC TGCTGG-3'.

Histology of the intestine and LCT activity analysis. Rat intestines were immersed in $4 \%$ paraformaldehyde for $4 \mathrm{~h}$ and transferred to $70 \%$ ethanol. Biopsy material was placed in processing cassettes, dehydrated through an alcohol gradient series and embedded in paraffin wax blocks. Before staining, 5 - $\mu \mathrm{m}$-thick tissue sections were dewaxed in xylene, rehydrated through decreasing concentrations of ethanol, washed in PBS and stained with H\&E. After staining, the sections were dehydrated in increasing concentrations of ethanol and xylene. Images were collected using a DM750 microscope (Leica, Germany). Rat intestine was obtained for LCT activity analysis using a Rat LCT ELISA Kit (Cusabio, Wuhan, China).
Statistics. Statistical analysis was performed using the SPSS 16.0 software (SPSS, Inc., Chicago, IL, USA). Normality of distributions were tested by Shapiro-Wilk test. Homogeneity of variance was determined by Levene's test. Differences between groups were examined using Student's $t$-test, chi-square test and one-way ANOVA followed by post hoc Tukey test, two-way ANOVA.

PCA, PLS-DA and OPLS-DA were used for metabolic profiling analysis. The Mann-Whitney-Wilcoxon test was used to analyse metabolites that differed in abundance between groups corrected for the FDR. The $P$-values from the enrichment analysis were adjusted for multiple testing of FDR.

The Wilcoxon rank-sum test, LefSe, ANOSIM and Metastats ${ }^{59}$ were used for the $16 \mathrm{~S}$ ribosomal RNA and metagenomic sequencing analyses. The different abundance of microbiota and enzymes between groups were analysed by Fisher's exact test with FDR adjustment. Correlations between the contents of Lactobacillus and D-galactose were identified by Pearson correlation analysis.

All $P$-values were two-sided, and $P<0.05$ was considered statistically significant. All data are expressed as mean $\pm \mathrm{SD}$.

Data availability. All data generated or analysed during this study are available from the authors upon reasonable request. All 16S rRNA sequences have been deposited to SRA database under the accession codes : SRP140781.

Received: 25 July 2017 Accepted: 27 April 2018

Published online: 22 May 2018

\section{References}

1. Bass, J. L. et al. The effect of chronic or intermittent hypoxia on cognition in childhood: a review of the evidence. Pediatrics 114, 805-816 (2004).

2. Pierson, D. J. Pathophysiology and clinical effects of chronic hypoxia. Respir Care 45, 39-51 (2000); discussion 51-33.

3. Nombela-Arrieta, C. \& Silberstein, L. E. The science behind the hypoxic niche of hematopoietic stem and progenitors. Hematol. Am. Soc. Hematol. Educ. Program 2014, 542-547 (2014).

4. Hare, J. M. et al. A randomized, double-blind, placebo-controlled, doseescalation study of intravenous adult human mesenchymal stem cells (prochymal) after acute myocardial infarction. J. Am. Coll. Cardiol. 54, 2277-2286 (2009)

5. Chen, S. L. et al. Effect on left ventricular function of intracoronary transplantation of autologous bone marrow mesenchymal stem cell in patients with acute myocardial infarction. Am. J. Cardiol. 94, 92-95 (2004).

6. Shin'oka, T. et al. Midterm clinical result of tissue-engineered vascular autografts seeded with autologous bone marrow cells. J. Thorac. Cardiovasc. Surg. 129, 1330-1338 (2005).

7. Le Blanc, K. et al. Treatment of severe acute graft-versus-host disease with third party haploidentical mesenchymal stem cells. Lancet 363, 1439-1441 (2004).

8. Le Blanc, K. et al. Mesenchymal stem cells for treatment of steroid-resistant, severe, acute graft-versus-host disease: a phase II study. Lancet 371, 1579-1586 (2008)

9. Jitschin, R. et al. Alterations in the cellular immune compartment of patients treated with third-party mesenchymal stromal cells following allogeneic hematopoietic stem cell transplantation. Stem Cells 31, 1715-1725 (2013).

10. Ide, C., Nakano, N. \& Kanekiyo, K. Cell transplantation for the treatment of spinal cord injury - bone marrow stromal cells and choroid plexus epithelial cells. Neural Regen. Res. 11, 1385-1388 (2016).

11. Abe, K. et al. Stem cell therapy for cerebral ischemia: from basic science to clinical applications. J. Cereb. Blood Flow. Metab. 32, 1317-1331 (2012).

12. Kuroda, S. Bone marrow stromal cell transplantation for ischemic stroke -- its multi-functional feature. Acta Neurobiol. Exp. (Wars.) 73, 57-65 (2013).

13. Hwa, R. C. Rapidity-interval dependence of multiplicity fluctuation. Phys. Rev. D Part. Fields 37, 2451-2457 (1988).

14. Hare, J. M. et al. Comparison of allogeneic vs autologous bone marrowderived mesenchymal stem cells delivered by transendocardial injection in patients with ischemic cardiomyopathy: the POSEIDON randomized trial. JAMA 308, 2369-2379 (2012).

15. Gupta, P. K., Das, A. K., Chullikana, A. \& Majumdar, A. S. Mesenchymal stem cells for cartilage repair in osteoarthritis. Stem Cell Res. Ther. 3, 25 (2012).

16. Williams, A. R. \& Hare, J. M. Mesenchymal stem cells: biology, pathophysiology, translational findings, and therapeutic implications for cardiac disease. Circ. Res. 109, 923-940 (2011).

17. Mohyeldin, A., Garzon-Muvdi, T. \& Quinones-Hinojosa, A. Oxygen in stem cell biology: a critical component of the stem cell niche. Cell. Stem. Cell. 7, 150-161 (2010).

18. Santisteban, M. M., Kim, S., Pepine, C. J. \& Raizada, M. K. Brain-gut-bone marrow axis: implications for hypertension and related therapeutics. Circ. Res. 118, 1327-1336 (2016).

19. Tang, W. H., Kitai, T. \& Hazen, S. L. Gut microbiota in cardiovascular health and disease. Circ. Res. 120, 1183-1196 (2017). 
20. Luo, Y. et al. Microbiota from obese mice regulate hematopoietic stem cell differentiation by altering the bone niche. Cell. Metab. 22, 886-894 (2015).

21. Drew, L. Microbiota: reseeding the gut. Nature 540, S109-S112 (2016).

22. Ellis, C. L., Rutledge, J. C. \& Underwood, M. A. Intestinal microbiota and blue baby syndrome: probiotic therapy for term neonates with cyanotic congenital heart disease. Gut Microbes 1, 359-366 (2010).

23. Rehman, S. U., Shah, S. A., Ali, T., Chung, J. I. \& Kim, M. O. Anthocyanins reversed D-galactose-induced oxidative stress and neuroinflammation mediated cognitive impairment in adult rats. Mol. Neurobiol. 54, 255-271 (2017).

24. Corno, A. F., Milano, G., Samaja, M., Tozzi, P. \& von Segesser, L. K. Chronic hypoxia: a model for cyanotic congenital heart defects. J. Thorac. Cardiovasc. Surg. 124, 105-112 (2002)

25. Salva, S., Marranzino, G., Villena, J., Aguero, G. \& Alvarez, S. Probiotic Lactobacillus strains protect against myelosuppression and immunosuppression in cyclophosphamide-treated mice. Int. Immunopharmacol. 22, 209-221 (2014).

26. D’Ippolito, G., Diabira, S., Howard, G. A., Roos, B. A. \& Schiller, P. C. Low oxygen tension inhibits osteogenic differentiation and enhances stemness of human MIAMI cells. Bone 39, 513-522 (2006).

27. Liu, S. et al. 2,5-hexanedione induced apoptosis of rat bone marrow mesenchymal stem cells by reactive oxygen species. J. Occup. Health 58, 170-178 (2016).

28. Larsen, S. A., Kassem, M. \& Rattan, S. I. Glucose metabolite glyoxal induces senescence in telomerase-immortalized human mesenchymal stem cells. Chem. Cent. J. 6, 18 (2012).

29. Coelho, A. I., Berry, G. T. \& Rubio-Gozalbo, M. E. Galactose metabolism and health. Curr. Opin. Clin. Nutr. Metab. Care 18, 422-427 (2015).

30. Suda, T., Takubo, K. \& Semenza, G. L. Metabolic regulation of hematopoietic stem cells in the hypoxic niche. Cell Stem Cell 9, 298-310 (2011).

31. Tiziani, S. et al. Metabolomics of the tumor microenvironment in pediatric acute lymphoblastic leukemia. PLoS ONE 8, e82859 (2013).

32. Shen, Y. et al. Glutamine synthetase plays a role in D-galactose-induced astrocyte aging in vitro and in vivo. Exp. Gerontol. 58, 166-173 (2014).

33. Hadzi-Petrushev, N., Stojkovski, V., Mitrov, D. \& Mladenov, M. D-galactose induced changes in enzymatic antioxidant status in rats of different ages. Physiol. Res. 64, 61-70 (2015).

34. Nam, S. M. et al. Valeriana officinalis extract and its main component, valerenic acid, ameliorate $\mathrm{D}$-galactose-induced reductions in memory, cell proliferation, and neuroblast differentiation by reducing corticosterone levels and lipid peroxidation. Exp. Gerontol. 48, 1369-1377 (2013).

35. $\mathrm{Li}, \mathrm{N}$. et al. D-galactose induces necroptotic cell death in neuroblastoma cell lines. J. Cell. Biochem. 112, 3834-3844 (2011).

36. Yu, Y. et al. Fibroblast growth factor (FGF21) protects mouse liver against Dgalactose-induced oxidative stress and apoptosis via activating Nrf2 and PI3K/ Akt pathways. Mol. Cell. Biochem. 403, 287-299 (2015).

37. Lu, T. \& Finkel, T. Free radicals and senescence. Exp. Cell Res. 314, 1918-1922 (2008).

38. Cao, C. et al. AMP-activated protein kinase contributes to UV- and H2O2induced apoptosis in human skin keratinocytes. J. Biol. Chem. 283, 28897-28908 (2008).

39. Fulle, S. et al. Stem cell ageing and apoptosis. Curr. Pharm. Des. 18, 1694-1717 (2012).

40. Lee, H. C. \& Wei, Y. H. Oxidative stress, mitochondrial DNA mutation, and apoptosis in aging. Exp. Biol. Med. 232, 592-606 (2007).

41. Chistiakov, D. A., Sobenin, I. A., Revin, V. V., Orekhov, A. N. \& Bobryshev, Y. $\mathrm{V}$. Mitochondrial aging and age-related dysfunction of mitochondria. Biomed. Res. Int. 2014, 238463 (2014).

42. Andersen, J. M. et al. Transcriptional and functional analysis of galactooligosaccharide uptake by lacS in Lactobacillus acidophilus. Proc. Natl. Acad. Sci. USA 108, 17785-17790 (2011)

43. Almeida, C. C., Lorena, S. L., Pavan, C. R., Akasaka, H. M. \& Mesquita, M. A Beneficial effects of long-term consumption of a probiotic combination of Lactobacillus casei Shirota and Bifidobacterium breve Yakult may persist after suspension of therapy in lactose-intolerant patients. Nutr. Clin. Pract. 27, 247-251 (2012).

44. Chow, J., Lee, S. M., Shen, Y., Khosravi, A. \& Mazmanian, S. K. Host-bacterial symbiosis in health and disease. Adv. Immunol. 107, 243-274 (2010).

45. Das, J. K. et al. Vaccines for the prevention of diarrhea due to cholera, shigella, ETEC and rotavirus. BMC Public. Health 13 (Suppl 3), S11 (2013).

46. Sassone-Corsi, M. et al. Microcins mediate competition among Enterobacteriaceae in the inflamed gut. Nature 540, 280-283 (2016).

47. Meehan, C. J. \& Beiko, R. G. A phylogenomic view of ecological specialization in the Lachnospiraceae, a family of digestive tract-associated bacteria. Genome Biol. Evol. 6, 703-713 (2014)

48. Kamo, T. et al. Dysbiosis and compositional alterations with aging in the gut microbiota of patients with heart failure. PLoS ONE 12, e0174099 (2017)
49. Moreno-Indias, I. et al. Intermittent hypoxia alters gut microbiota diversity in a mouse model of sleep apnoea. Eur. Respir. J. 45, 1055-1065 (2015).

50. $\mathrm{Xu}, \mathrm{C}$. L. et al. Protective effect of glutamine on intestinal injury and bacterial community in rats exposed to hypobaric hypoxia environment. World J. Gastroenterol. 20, 4662-4674 (2014).

51. Fedorova, T. A. et al. [The stomach and duodenum condition in patients with chronic obstructive lung diseases]. Klin. Med. (Mosk.) 81, 31-33 (2003).

52. Rastall, R. A. et al. Modulation of the microbial ecology of the human colon by probiotics, prebiotics and synbiotics to enhance human health: an overview of enabling science and potential applications. FEMS Microbiol. Ecol. 52, 145-152 (2005).

53. Wilck, N. et al. Salt-responsive gut commensal modulates TH17 axis and disease. Nature 551, 585-589 (2017).

54. Matter, R. M. et al. Determinants of platelet count in pediatric patients with congenital cyanotic heart disease: role of immature platelet fraction. Congenit. Heart Dis. 13, 118-123 (2018)

55. Zhu, W., Chen, J., Cong, X., Hu, S. \& Chen, X. Hypoxia and serum deprivation-induced apoptosis in mesenchymal stem cells. Stem Cells 24, 416-425 (2006).

56. Xin, Y. et al. Aging adversely impacts biological properties of human bone marrow-derived mesenchymal stem cells: implications for tissue engineering heart valve construction. Artif. Organs 34, 215-222 (2010).

57. Pittenger, M. F. et al. Multilineage potential of adult human mesenchymal stem cells. Science 284, 143-147 (1999).

58. Harrison, J. S., Rameshwar, P., Chang, V. \& Bandari, P. Oxygen saturation in the bone marrow of healthy volunteers. Blood 99, 394 (2002).

59. White, J. R., Nagarajan, N. \& Pop, M. Statistical methods for detecting differentially abundant features in clinical metagenomic samples. PLoS Comput. Biol. 5, e1000352 (2009).

\section{Acknowledgements}

This study was supported by the National Science Fund for Distinguished Young Scholars (81525002), National Natural Science Foundation of China (81430006 and 81400186) and Professor in Peking Union Medical College.

\section{Author contributions}

H.Z. conceived and supervised the study. Y.Y., Z.M., T.W. and F.Y. collected the patient samples. J.X., Y.Y. and M.X. performed the data analysis for metabolomics, $16 \mathrm{~S}$ ribosomal RNA sequencing and metagenomic sequencing. J.X., Y.Y. and M.X. performed the cell study. J.X. and Y.L. performed the animal study. J.X., Y.Y., C.M. and Q.Z. prepared the manuscript draft. J.X. and H.Z. revised the manuscript and provided extensive discussions. All authors participated in discussion and editing of the manuscript.

\section{Additional information}

Supplementary Information accompanies this paper at https://doi.org/10.1038/s41467 018-04453-9.

Competing interests: The authors declare no competing interests.

Reprints and permission information is available online at http://npg.nature.com/ reprintsandpermissions/

Publisher's note: Springer Nature remains neutral with regard to jurisdictional claims in published maps and institutional affiliations.

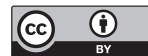

Open Access This article is licensed under a Creative Commons Attribution 4.0 International License, which permits use, sharing, adaptation, distribution and reproduction in any medium or format, as long as you give appropriate credit to the original author(s) and the source, provide a link to the Creative Commons license, and indicate if changes were made. The images or other third party material in this article are included in the article's Creative Commons license, unles indicated otherwise in a credit line to the material. If material is not included in the article's Creative Commons license and your intended use is not permitted by statutory regulation or exceeds the permitted use, you will need to obtain permission directly from the copyright holder. To view a copy of this license, visit http://creativecommons.org/ licenses/by/4.0/

\section{(c) The Author(s) 2018}

